\title{
HEAVY-ELEMENT ABUNDANCES IN SOLAR ENERGETIC PARTICLE EVENTS
}

\author{
D. V. Reames and C. K. $\mathrm{Ng}^{1}$ \\ Code 661 NASA/Goddard Space Flight Center, Greenbelt, MD 20771 \\ reames@milkyway.gsfc.nasa.gov, cheeng@milkyway.gsfc.nasa.gov
}

Accepted by Astrophysical Journal

${ }^{1}$ Also Department of Astronomy, University of Maryland, College Park, MD 20742 
ABSTRACT

We survey the relative abundances of elements with $1 \leq Z \leq 82$ in solar energetic particle (SEP) events observed at $2-10 \mathrm{MeV}$ amu $^{-1}$ during nearly 9 years aboard the Wind spacecraft, with special emphasis on enhanced abundances of elements with $Z \geq 34$. Abundances of $\mathrm{Fe} / \mathrm{O}$ again show a bimodal distribution with distinct contributions from impulsive and gradual SEP events as seen in earlier solar cycles. Periods with greatly enhanced abundances of $(50 \leq \mathrm{Z} \leq 56) / \mathrm{O}$, like those with enhanced ${ }^{3} \mathrm{He} /{ }^{4} \mathrm{He}$, fall prominently in the Fe-rich population of the impulsive SEP events. In a sample of the 39 largest impulsive events, 25 have measurable enhancements in (50 $Z \leq 56) / O$ and $(76 \leq Z \leq 82) / 0$, relative to coronal values, ranging from $\sim 100$ to 10,000 . By contrast, in a sample of 45 large gradual events the corresponding enhancements vary from $\sim 0.2$ to 20 . However, the magnitude of the heavy-element enhancements in impulsive events is less striking than their strong correlation with the Fe spectral index and flare size, with the largest enhancements occurring in flares with the steepest $\mathrm{Fe}$ spectra, the smallest $\mathrm{Fe}$ fluence, and the lowest $X$-ray intensity, as reported here for the first time Thus it seems that small events with low energy input can produce only steep spectra of the dominant species but accelerate rare heavy elements with great efficiency, probably by selective absorption of resonant waves in the flare plasma. With increased energy input, enhancements diminish, as heavy ions are depleted, and spectra of the dominant species harden.

Subject headings: acceleration of particles -shock waves-Sun: abundances-Sun: corona1 mass ejections (CMEs) - Sun: flares- Sun: particle emission 


\section{INTRODUCTION}

Relative abundances of elements and isotopes have proven to be a rich source of information on the origin and history of populations of energetic ions we have encountered throughout the heliosphere (e.g. Reames 1999). In the solar energetic particle (SEP) events, abundances have aided in distinguishing two underlying mechanisms of particle acceleration that contribute to "gradual" and "impulsive" SEP events (see reviews Reames 1990, 1995b, 1999, 2002; Kahler 1992, 1994, 2001; Gosling 1993; Lee 1997; Tylka 2001). In the large gradual SEP events, abundances of elements up to $\mathrm{Fe}$ at a few $\mathrm{MeV} \mathrm{amu}{ }^{-1}$ are, on average, similar to those of the solar corona and solar wind (Meyer 1985; Reames 1995a, 1999) from which ions are accelerated by shock waves driven out from the sun by coronal mass ejections (CMEs). In contrast, the impulsive SEP events associated with solar flares have 1000 -fold enhancements of ${ }^{3} \mathrm{He} /{ }^{4} \mathrm{He}$ and enhancements in heavier elements that increase with decreasing charge-to-mass ratio $Q / A$ of the ions at coronal temperatures (e.g. Reames, Meyer, \& von Rosenvinge 1994). The pattern of enhancements in impulsive SEP events is generally believed to result from resonant waveparticle interactions in the turbulent flare plasma (Fisk 1978; Temerin \& Roth 1992; Miller \& Viñas 1993; Roth \& Temerin 1997; Miller 1998). In some large SEP events, however, intermediate abundances may result when remnant suprathermal ions from impulsive events contribute to the material accelerated by CME-driven shock waves (Mason et al 1999; Tylka et al 2001; Desai et al 2003). In addition, the effects of particle transport from the source can cause abundances to vary with particle energy and with space and time during gradual events (Ng, Reames, \& Tylka 2003).

For elements with atomic number $Z \geq 34$, solar abundances decrease precipitously by as much as $\sim 6$ to 8 orders of magnitude below that of O (Grevesse \& Sauval 1998), and early instruments were unable to collect enough ions to make regular measurements of abundances in this region. The earliest measurements of heavy-element abundances in SEP events were made using particle tracks collected in a glass window of the Apollo 16 command module (Shirk \& Price 1972). In recent years, however, regular measurements of abundances of the elements throughout the periodic table above $\mathrm{Fe}$ have begun to contribute to the study of SEP events (Reames 2000; Reames, Ng, \& Tylka 2001; Tylka et al. 2002; Mason et al 2004). For impulsive events, $Q / A$-dependent enhancements continue into the region of heavy elements, producing $\sim 1000$-fold enhancements for $(Z \geq 50) / \mathrm{O}$. For gradual events, however, ions with $Z \geq 34$ often have coronal abundances or only modest enhancements that follow a temporal behavior similar to that of $\mathrm{Fe} / \mathrm{O}$ and show high-energy spectral rollovers with the same $Q / A$-dependence as the elements up through $\mathrm{Fe}$ (Reames et al. 2001). Most of the recent measurements of ions with $34 \leq Z \leq 82$ mentioned above have been made in the 3.3 to $10 \mathrm{MeV} \mathrm{amu}^{-1}$ region with the large-geometry Low-Energy Matrix Telescope (LEMT) on the Wind spacecraft that we discuss herein. Recently, however, time-of-flight-based measurements in the 0.1 to $1 \mathrm{MeV} a m u^{-1}$ region showing similar heavy-element enhancements in ${ }^{3} \mathrm{He}$-rich events have been reported by Mason et al. (2004).

The previous heavy-element observations from LEMT were reported for limited time periods or in selected SEP events. In this paper we extend those results significantly by surveying all such measurements from 1994 November 4 through 2003 September 28. This allows us to study the statistical properties of heavy element abundances in a large 
sample of SEP events of various kinds. Properties of the LEMT telescope have been described by von Rosenvinge et al. (1995) and its response to heavy elements has been described in detail by Reames (2000).

\section{THE SYNOPTIC VIEW.}

A classical way to provide an overall view of the systematic variations of element and isotopic abundances has been to divide the entire sample period into daily averages and to plot the pattern of intensities of one species against another on a day-to-day basis. This technique allows one to view the full sweep of abundance variations with no bias as to the selection of "events" for study; each day is an "event". Reames (1988) made plots of daily-averaged Fe vs. $\mathrm{O}$ at $\sim 2 \mathrm{MeV} \mathrm{amu}^{-1}$ and found a bimodal distribution corresponding to the presence of impulsive and gradual SEP events. Distinguishing the points in the plot of $\mathrm{Fe}$ vs. $\mathrm{O}$ by their corresponding ${ }^{3} \mathrm{He} /{ }^{4} \mathrm{He}$, proton/electron, or $\mathrm{He} / \mathrm{H}$ ratios, the underlying properties of the contributing impulsive and gradual events were further identified.

Owing to the greater sensitivity of LEMT, we have divided the present data set into 8-hour intervals and show in Figure $1 \mathrm{Fe} v$ s. O intensities for each of those intervals in the $\sim 9$-year study period. In the upper panel of the figure, the color and size of the symbols varies with the ${ }^{3} \mathrm{He} /{ }^{4} \mathrm{He}$ ratio at $2.1-2.5 \mathrm{MeV}$ amu ${ }^{-1}$ during each interval, while symbols in the lower panel depend upon the $(50 \leq \mathrm{Z} \leq 56) / \mathrm{O}$ abundance ratio at $3.3-10 \mathrm{MeV}$ $\mathrm{amu}^{-1}$. The approximate intensity of background anomalous cosmic ray (ACR) O during solar minimum is marked on the panels. This represents an approximate lower bound for the O intensity for the years 1994-1998 (see Reames \& McDonald 2003) during which many small SEP events occur. Each panel in Figure 1 contains about 4900 points.

In the upper panel of Figure 1, it is not difficult to follow the locus of the distributions we identify with impulsive and gradual SEP events at or slightly above the lines of $\mathrm{Fe} / \mathrm{O}=1$ and 0.1 , respectively. The ${ }^{3} \mathrm{He}$-rich intervals cluster near the line with $\mathrm{Fe} / \mathrm{O}=1$ at moderate intensities but are deflected below that line at very low intensities when background from $\mathrm{ACR} O$ is present to depress $\mathrm{Fe} / \mathrm{O}$ from the impulsive events. At higher intensities of $\mathrm{Fe}$ or $\mathrm{O},{ }^{3} \mathrm{He} /{ }^{4} \mathrm{He}$ in impulsive events decreases to values below 0.2 that are not easily measured by LEMT because of its limited resolution (Reames et al. 1997). However, the track of impulsive event intervals seems to end where the intensities of $\mathrm{Fe}$ or $\mathrm{O}$ reach $\sim 10^{-2}$ particles $\mathrm{cm}^{-2} \mathrm{sr}^{-1} \mathrm{~s}^{-1} \mathrm{MeV}^{-1}$ amu. Intervals during gradual events are distributed about the line $\mathrm{Fe} / \mathrm{O}=0.1$ at low and moderate intensities, but generally rise above $\mathrm{Fe} / \mathrm{O}=0.1$ at high intensities; many of these high-intensity intervals occur early during large SEP events when $\mathrm{Fe} / \mathrm{O}$ is elevated by transport-dominated effects (e.g. Tylka, Reames, \& Ng 1999; Ng, Reames, \& Tylka 1999; Reames, Ng, \& Tylka 2000), including those in the large "Bastille-day" event of 2000 July 14 (Reames, $\mathrm{Ng}$, \& Tylka 2001). Note that, at the very lowest Fe intensities, a few of the 8-hr intervals distributed near $\mathrm{Fe} / \mathrm{O} \sim 0.1$ actually correspond to ions from corotating interacting regions for which abundances are also un-enhanced (see e.g. Reames 1999).

In the lower panel of Figure $1,(50 \leq \mathrm{Z} \leq 56) / \mathrm{O}$ ratios highlight the points. Intervals with large values of this ratio are seen to cluster along the track of $\mathrm{Fe} / \mathrm{O}=1$. At the lowest intensities, of course, elements with $50 \leq Z \leq 56$ can only be seen when these ions are greatly enhanced relative to the coronal $(50 \leq \mathrm{Z} \leq 56) / \mathrm{O}$ ratio taken as $2.90 \times 10^{-6}$ (Grevesse 
\& Sauval 1998; see Reames 2000). Near the top of the impulsive region where Fe or O reach $\sim 10^{-2}$ particles $\mathrm{cm}^{-2} \mathrm{sr}^{-1} \mathrm{~s}^{-1} \mathrm{MeV}^{-1}$ amu, near-coronal abundances of $(50 \leq \mathrm{Z} \leq 56) / \mathrm{O}$ would be measurable, but 100 - and 1000 -fold enhancements of $(50 \leq Z \leq 56) / O$ are seen. These large enhancements along the impulsive track contrast with nearly coronal values in the gradual region nearby. Occasional instances of significantly enhanced $(50 \leq \mathrm{Z} \leq 56) / \mathrm{O}$ in the gradual population $(\mathrm{Fe} / \mathrm{O} \sim 0.1)$ at intermediate intensities usually correspond to intervals where both impulsive and gradual events contribute, as we shall see in Section 4 below.

Superficially it may seem that ${ }^{3} \mathrm{He}$ - and $\mathrm{Z} \geq 50$ ion enhancements occupy nearly exclusive regions of the $\mathrm{Fe} / \mathrm{O} \sim$, impulsive-event track. However, instrument sensitivity limits the observation of even greatly enhanced $Z \geq 50$ ions in small events and ${ }^{3} \mathrm{He} /{ }^{4} \mathrm{He}$ ratios decrease in large events, probably because the supply of ${ }^{3} \mathrm{He}$ ions in the flare volume is becoming exhausted (Reames 1999). These two facts conspire to limit the observational overlap of ${ }^{3} \mathrm{He}$ - and $Z \geq 50$ ion enhancements. We believe that the apparent separation of impulsive events with ${ }^{3} \mathrm{He}$ - and $\mathrm{Z} \geq 50$ enhancements is entirely instrumental.

Finally, we point out that the 8-hr averaging of the data in Figure 1 distorts the relative probability of impulsive and gradual events. Gradual events, lasting several days, are sampled several times while more than one impulsive event may contribute to a single point in the figure.

\section{IMPULSIVE EVENTS}

\subsection{Preliminary Impulsive Event Selection}

To place the heavy-element abundances in the general context of impulsive events, we have begun by scanning hourly-averaged data to select a preliminary list of events using two different criteria: 1) events where ${ }^{3} \mathrm{He} /{ }^{4} \mathrm{He}>0.5$ and intensities of ${ }^{3} \mathrm{He}$ or ${ }^{4} \mathrm{He}$ exceed $10^{-4}$ particles $\mathrm{cm}^{-2} \mathrm{sr}^{-1} \mathrm{~s}^{-1} \mathrm{MeV}^{-1}$ amu, and 2) events where $\mathrm{Fe} / \mathrm{O}>0.5$ and intensities of Fe intensities exceed $10^{-5}$ particles $\mathrm{cm}^{-2} \mathrm{sr}^{-1} \mathrm{~s}^{-1} \mathrm{MeV}^{-1}$ amu. The second criterion for impulsive events is clearly guided by the distributions of particle abundances shown in Figure 1. A total of 133 events were found, 80 met the ${ }^{3} \mathrm{He}$-rich criteria, 82 met the Fe-rich criteria and 29 met both criteria. Note that ${ }^{3} \mathrm{He}$-rich events can occur in intervals that need not contribute to Figure 1 because the events are too small to have measurable $\mathrm{Fe}$ and $\mathrm{O}$ intensities. Relaxing the criteria on the intensities of ${ }^{3} \mathrm{He}$ and ${ }^{4} \mathrm{He}$ or their ratio would have produced hundreds of candidate events. However, determining that intensity increases are truly new event onsets becomes difficult for smaller events, so we have used conservative criteria.

Intensity-time profiles for events with a variety of properties are compared in Figure 2. Events 1 and 2 in the figure are ${ }^{3} \mathrm{He}$-rich $\left({ }^{3} \mathrm{He} /{ }^{4} \mathrm{He} \sim 10\right)$ events of moderate intensity for which the intensities of $\mathrm{Fe}$ and $\mathrm{O}$ are too low for measurement. The latter is completely obscured by the ACR $O$ that runs across the panel. Events 3 and 4 are somewhat larger though they occur in a high background of $\mathrm{H}$ and ${ }^{4} \mathrm{He}$ from a gradual event much earlier. These events have similar intensities of ${ }^{3} \mathrm{He}$, but Event 4 has more ${ }^{4} \mathrm{He}$ and clear increases in $\mathrm{O}$ and $\mathrm{Fe}$. Event 5 is similar to Event 4, but shows a small increase of 
associated heavy elements. Events 6 and 7 have higher intensities of ${ }^{4} \mathrm{He}, \mathrm{Fe}$ and $\mathrm{O}$ and significant numbers of heavy elements. ${ }^{3} \mathrm{He} /{ }^{4} \mathrm{He}$ is substantially reduced in both events, although it is measurable in Event 7. Events 8 and 9 have similar intensities of $\mathrm{H},{ }^{4} \mathrm{He}, \mathrm{O}$ and $\mathrm{Fe}$, but Event 9 has a substantial number of heavy ions and measurable ${ }^{3} \mathrm{He}$. Events 10-13 are a series of large impulsive events that sometimes occur when the spacecraft is inside the CME following a gradual event and is thus magnetically well-connected to an active region where flares are likely (see Reames 2000 for a similar example).

The rate of occurrence of impulsive events increases with the solar cycle (e.g. Reames et al. 1994). However, near solar maximum, these small events are obscured by the presence of large gradual events. During the years 2000, 2001, and 2002, for example, the 2.6-3.2 MeV amu ${ }^{-1} \mathrm{O}$ intensity is above $10^{-4}$ particles $\mathrm{cm}^{-2} \mathrm{sr}^{-1} \mathrm{~s}^{-1} \mathrm{MeV}^{-1}$ amu for $37 \%$ of the time, making it difficult to see and measure $\mathrm{Fe}$ and $\mathrm{O}$ in impulsive events with this intensity or less. During the same 3 years the $2.0-2.4 \mathrm{MeV}$ amu${ }^{-1}{ }^{4} \mathrm{He}$ intensity is above $10^{-3}$ particles $\mathrm{cm}^{-2} \mathrm{sr}^{-1} \mathrm{~s}^{-1} \cdot \mathrm{MeV}^{-1}$ amu for $68 \%$ of the time, making the observation of small ${ }^{3} \mathrm{He}$-rich events even more difficult. During these years, the 2.1-2.5 MeV H intensity is above the extremely high value of 1 particle $\mathrm{cm}^{-2} \mathrm{sr}^{-1} \mathrm{~s}^{-1} \mathrm{MeV}^{-1}$ for $43 \%$ of the time.

\section{2 "Large" Impulsive Events - Abundances}

Clearly, as seen from Figure 1, heavy ions are most likely to be found in the most intense Fe-rich impulsive SEP events. Therefore, from the original sample of Fe-rich impulsive events we select a subset of "large" impulsive events with Fe intensity above $10^{-4}$ particles $\mathrm{cm}^{-2} \mathrm{sr}^{-1} \mathrm{~s}^{-1} \mathrm{MeV}^{-1}$ amu. Properties of the 39 large impulsive events selected are shown in Table 1. Of the 39 events, 33 events have $Z \geq 34$ ions and 25 events have $Z \geq 50$ ions. (All of the 14 events with Fe intensity above $10^{-3}$ particles $\mathrm{cm}^{-2} \mathrm{sr}^{-1} \mathrm{~s}^{-1}$ $\mathrm{MeV}^{-1}$ amu have heavy elements.)

In Figure 3 we show the time-integrated heavy-element enhancements as a function of the corresponding enhancement of $\mathrm{Fe} / \mathrm{O}$ for the sample of 39 large impulsive events defined above. The coronal abundance ratios for Fe/O, $(34 \leq \mathrm{Z} \leq 40) / \mathrm{O},(50 \leq \mathrm{Z} \leq 56) / \mathrm{O}$, and $(76 \leq \mathrm{Z} \leq 82) / \mathrm{O}$ are taken as $0.134,2.22 \times 10^{-5}, 2.90 \times 10^{-6}$, and $1.01 \times 10^{-6}$, respectively. The enhancement factors cluster around $\sim 10, \sim 100, \sim 1000$, and $\sim 1000$ for the four species, respectively, and there is little evidence of any correlated behavior when we consider only impulsive events. This is consistent with the poor correlations found in the event-toevent abundance variations for elements up to Fe (Reames et al. 1994).

To summarize the heavy element abundances in impulsive events, we have averaged the element abundances over all of the 39 "large" impulsive-event periods, whether they had heavy elements or not. The average abundance enhancements of the elements in these events are shown in Figure 4 as solid circles as functions of $Z$ and of $Q / A$ using the equilibrium $Q$ at a temperature of $\sim 3 \mathrm{MK}$. Open circles in the Figure 4 supplement these measurements with the average enhancements of other elements determined in the same energy range in earlier studies of impulsive events (Reames et al. 1994; Reames 1995a, 1999). Ionization states at a coronal temperature of $\sim 3 \mathrm{MK}$ have been shown to organize element abundances in impulsive events (Reames et al. 1994). Values of $Q$ at a temperature of $\sim 3 \mathrm{MK}$ for the elements were found from Arnaud \& Rothenflug (1985) for $Z<26$, from Arnaud and Raymond (1992) for $Z=26$ and from Post et al (1977) for $Z>26$. 


\section{2 "Large" Impulsive Events - Spectra}

Figure 5 shows energy spectra for ${ }^{4} \mathrm{He}, \mathrm{C}, \mathrm{O}, \mathrm{Ne}, \mathrm{Si}, \mathrm{Fe}$, and for ions with $34 \leq \mathrm{Z} \leq 40$ and $50 \leq Z \leq 56$ in 3 of the large impulsive SEP events that have significant intensities of heavy elements. The spectra of different elements can be seen to differ in slope within a given event and to some extent they depart from a power law behavior. This is not surprising since theoretical spectra (Miller 1998) and theoretical fits to observations (Mason et al. 2003) have shown complex behavior. However, to illustrate the range of variation, we find least-squares power-law spectral indices for ${ }^{4} \mathrm{He}, \mathrm{C}, \mathrm{O}, \mathrm{Ne}, \mathrm{Si}, \mathrm{Fe}$ and $34 \leq \mathrm{Z} \leq 40$ of $-2.59 \pm 0.18,-3.10 \pm 0.12,-3.17 \pm 0.12,-3.33 \pm 0.16,3.04 \pm 0.13,-2.77 \pm 0.16$, and $2.17 \pm 0.71$, respectively in the 2001 April 14 event. The corresponding spectral indices for the 2002 August 20 event are $-2.51 \pm 0.16,-2.15 \pm 0.08,-1.88 \pm 0.06,-1.93 \pm 0.07$, $1.91 \pm 0.06,-1.80 \pm 0.11$, and $-1.50 \pm 0.33$. A difference in the spectra of different elements means that their relative abundances, and enhancements, will vary with energy. For these two events there is a tendency for $(34 \leq \mathrm{Z} \leq 40) / \mathrm{O}$ to rise almost linearly with energy, although the errors in this determination are large.

The sample spectra above show considerable variation in spectral slope among the species shown. In particular, they suggest that the spectrum of Fe can be harder than that of $\mathrm{O}$ in an event, and perhaps the spectra of heavier ions can be harder than the spectra of Fe. Measurement statistics do not allow us to study the systematic behavior of heavy element spectra, but we can compare the spectral indices of $\mathrm{Fe}$ and $\mathrm{O}$ in all 39 large impulsive events (see Table 1) as shown in Figure 6. Most events fall below the dashed diagonal line in the figure showing that Fe spectra are systematically harder than $\mathrm{O}$ spectra. As the spectra become softer the points fall increasingly below the diagonal line, and the difference between $\mathrm{Fe}$ and $\mathrm{O}$ becomes greater. This trend is also shown by the solid least-squares fit line.

Armed with this information on the impulsive event spectra, we plot in Figure 7 the enhancement in $(34 \leq \mathrm{Z} \leq 40) / \mathrm{O},(50 \leq \mathrm{Z} \leq 56) / \mathrm{O}$, and $(76 \leq \mathrm{Z} \leq 82) / \mathrm{O}$ abundances, relative to the corona, as a function of the Fe spectral index for the large impulsive events. For clarity, we have omitted from the figure events with abundances that are only upper limits or have large errors. A high degree of correlation is seen in the figure and the correlation coefficients increase as $Z$ of the heavy elements increases. Poorer correlations are found when the spectral index of $O$ replaces that of $F e$.

Anticipating that steep spectra might be associated with small events with reduced energy input, we plot in the left panel of Figure 8 events with well-determined enhancement in $(50 \leq \mathrm{Z} \leq 56) / \mathrm{O} v \mathrm{~s}$. the Fe fluence. Of course, the fluence will depend upon how magnetically well connected the events are and in a few cases the time integration of $\mathrm{Fe}$ is truncated by the onset of a new event. Nevertheless, small events have greater enhancements of $(50 \leq Z \leq 56) / O$. The enhancements are plotted $v s$. the NOAA/GOES soft $\mathrm{X}$-ray peak flux for each event in the right panel of Figure 8. Most of the events come from C- and M-class flares. Soft X-rays represent heating of the flare plasma, and is more sensitive to acceleration of electrons than ions. Nevertheless, a substantial correlation is found here as well. Reames et al (1988) found a similar behavior for ${ }^{3} \mathrm{He} /{ }^{4} \mathrm{He}$ in impulsive events; ${ }^{3} \mathrm{He} /{ }^{4} \mathrm{He}$ decreased as hard and soft $\mathrm{X}$-ray fluxes and $\mathrm{Km}$ type III radio intensities increased. 


\section{GRADUAL EVENTS}

For comparison with the impulsive events described above, we have selected a sample of large gradual events. Guided by Figure 1, we chose as gradual all events in which the intensity of 2.6-3.2 MeV amu ${ }^{-1} \mathrm{O}$ exceeded $10^{-2}$ particles $\mathrm{cm}^{-2} \mathrm{sr}^{-1} \mathrm{~s}^{-1} \mathrm{MeV}^{-1}$ amu; 48 large events met this criterion. Properties of these events are shown in Table 2.

Figure 9 shows intensities of various ion species and enhancements relative to coronal abundances for $\mathrm{Fe} / \mathrm{O},(34 \leq \mathrm{Z} \leq 40) / \mathrm{O}$, and $(50 \leq \mathrm{Z} \leq 56) / \mathrm{O}$ as a function of time during three gradual events. These events, from CME sources at three different solar longitudes, show enhancements in $(34 \leq \mathrm{Z} \leq 40) / \mathrm{O}$ that are only modestly larger than those in $\mathrm{Fe} / \mathrm{O}$, and have a similar time dependence. Despite the high intensities of lighter ions in the large events in Figure 9, which exceed those of impulsive events by orders of magnitude (compare Figure 2), ions with $Z \geq 50$ are sufficiently rare that we cannot follow their time dependence. Data leading to similar conclusions has been shown in other gradual events (Reames 2000; Reames et al. 2001).

In Figure 10 we show the time-integrated heavy-element enhancements as a function of the enhancement of $\mathrm{Fe} / \mathrm{O}$ for the sample of 48 large gradual SEP events defined above. Here the average enhancement factors are quite modest, in contrast with those for impulsive events shown in Figure 3. The $(34 \leq \mathrm{Z} \leq 40) / \mathrm{O}$ enhancements shown in Figure 10 are correlated with those of $\mathrm{Fe} / \mathrm{O}$ with a correlation coefficient, $r=0.86$, and the leastsquares fit line passes close to the un-enhanced coronal abundances for both species. Strong correlation of element abundances in gradual SEP events is common (e.g. Reames 1995a, 1999). The heavier elements in Figure 10 are sufficiently rare, despite the high fluence of $\mathrm{Fe}$ ions, that correlations cannot be established. Upper limits on the enhancements of $Z \geq 34$ ions are suppressed in Figure 10, but are listed in Table 2; these limits confine the enhancements to very modest values. Analysis of the time evolution of the large gradual events suggest that some of the $Z \geq 50$ ions arrive late in the events, after the time of shock passage when they might come from small impulsive SEP events that are otherwise obscured by high intensities of elements with $Z \leq 26$ (an example occurs during the event of 2001 November 23 shown in Figure 9).

\section{DISCUSSION}

The overall pattern of the intensities of $\mathrm{Fe}$ and $\mathrm{O}$ and other species in the region of $\sim 1-10 \mathrm{MeV} \mathrm{amu}{ }^{-1}$ supports the earlier findings (Reames 1988) of a bimodal distribution reflecting both impulsive and gradual SEP events. The two classes of events are clearly present, although they are not fully resolved by $\mathrm{Fe} / \mathrm{O}$ alone. These event classes, originally distinguished by the time-scale of the associated soft X-ray events, have come to differentiate the effects of the physical mechanism of resonant stochastic acceleration in flares from that of shock acceleration at CME-driven shock waves (Reames 1999, 2002).

Variations of abundances with energy may make patterns, that are obvious in our energy region, become obscured in other regions. Differences in the spectral indices of different species that we see will cause abundance variations to be greatly magnified at higher energies. When we attempt to re-plot Figure 1 using $O$ and Fe intensities near 10 $\mathrm{MeV} \mathrm{amu} u^{-1}$, we find that the distinction between impulsive and gradual events becomes 
blurred and loses its bimodal character. Therefore, abundances measured above $10 \mathrm{MeV}$ $a m u^{-1}$ may be completely useless in distinguishing impulsive and gradual SEP events.

There are certainly mechanisms that could blur the resolution of impulsive and gradual events at other energies. Below $1 \mathrm{MeV} a \mathrm{mu}^{-1}$, abundances may be strongly affected by differing transport (e.g. Ng et al. 2003) and magnetic connections (Mazur et al. 2000) during the long transit times of many hours or days from the source to the observer. Above $10 \mathrm{MeV} \mathrm{amu}{ }^{-1}$, energy-dependent differences in the trapping and acceleration of ions in the source region must become important. Perhaps these same mechanisms cause the abundances to vary with energy as we observe. However, poor discrimination of impulsive and gradual events at other energies does not negate the bimodal abundance pattern that we have seen for 3 solar cycles in the region of a few $\mathrm{MeV}$ $a m u^{-1}$.

When we examine the heavy element events listed by Mason et al. (2004), we find that many of the events have low values of ${ }^{3} \mathrm{He} /{ }^{4} \mathrm{He}(<0.01)$ and $\mathrm{Fe} / \mathrm{O}(<0.5)$. We believe these are likely to be gradual events, although criteria may differ in the $0.1-1.0 \mathrm{MeV}$ $\mathrm{amu}^{-1}$ region. If we focus on the 9 events listed with positive measurements of $(m>100) / F e$ (and enhancement factors of 600-5000), all have $\mathrm{Fe} / \mathrm{O}>1$ (1.49-4.55) and are surely impulsive events. Of these, 5 are also found on our list of 39 large impulsive events described above; the remaining 4 events show little or no increase in $\mathrm{Fe}$ above 2 $\mathrm{MeV} \mathrm{amu}{ }^{-1}$, suggesting that these events have sufficiently steep spectra that they are too small to be seen at high energies. The Mason et al. (2004) list contains events with intensities of $\mathrm{Fe}>0.1$ ions $\mathrm{cm}^{-2} \mathrm{sr}^{-1} \mathrm{~s}^{-1} \mathrm{MeV}^{-1}$ amu above $100 \mathrm{keV} \mathrm{amu}^{-1}$ while our final list contains events with intensities of $\mathrm{Fe}>10^{-4}$ ions $\mathrm{cm}^{-2} \mathrm{sr}^{-1} \mathrm{~s}^{-1} \mathrm{MeV}^{-1}$ amu above $2 \mathrm{MeV}$ $a m u^{-1}$. Small events with differential energy spectra steeper than $\sim E^{-4}$ could be seen by Mason et al. (2004) but not by us. The 4 events on the Mason et al. (2004) list that are too steep to be seen by LEMT include the 3 highest values of $(m>100) / F e$ enhancement that they report, supporting our finding that the events with the steepest spectra have the greatest heavy element enhancements. The 5 events we have in common have $\mathrm{Fe}$ spectral indices between -1.8 and -3.4 .

Fe spectral indices of our 39 large impulsive events range from -1.8 to -4.4 with a mean value of -3.1 . There is a correlation $(r=0.81)$ between the $(50 \leq Z \leq 56) / O$ enhancement and the $\mathrm{Fe}$ spectral index so that events with steeper $\mathrm{Fe}$ spectra have greater $(50 \leq Z \leq 56) / O$ enhancements. Over the observed spectral range, enhancements vary from $\sim 10^{2}$ to $\sim 10^{4}$ and a similar range of enhancements is seen for $(76 \leq \mathrm{Z} \leq 82) / \mathrm{O}$. Differences in spectra are an essential factor in the enhancement of heavy elements. Furthermore, small events, as measured by the Fe fluence, have greater enhancements. The Fe spectral index is only weakly correlated $(r=0.46)$ with the Fe fluence for the 39 events.

Fe fluence is one measure of impulsive event size and soft X-ray flux is another; both suggest that small events with less energy input have the greatest heavy-element enhancements. A similar result was found for enhancements in ${ }^{3} \mathrm{He} /{ }^{4} \mathrm{He}$ (Reames et al 1988).

Qualitatively the heavy element and spectral behavior may be understood based upon the model of cascading waves (e.g. Miller 1998) in which waves generated at long wavelengths (low wave number $k$ ), by magnetic reconnection in a flare, cascade toward higher $k$, passing through resonance with ions of increasing gyrofrequency or $Q / A$ as they go. When the energy in waves is low, most of it can be absorbed by the ions at highest $Z$ 
(lowest $Q / A$ ) causing greater enhancements, but steeper spectra, because of the limited available energy, especially at lower Z. In larger flares more energy is available to produce harder spectra and wave absorption by the limited numbers of heavy ions is inadequate to stem the flood of cascading waves. Unfortunately, however, we do not yet have a quantitative model that explains the full range of observations.

The above arguments suggest that saturation in the magnitude of heavy element enhancements becomes important as the flare energy increases. In the flare plasma, there are only $\sim 2$ ions with $50 \leq Z \leq 56$ for every $10^{10} \mathrm{H}$ ions (Grevesse \& Sauval 1998). Ultimately, once all the $Z \geq 50$ ions in the flare volume have been accelerated, additional energy can only go into lighter ions so the enhancements begin to decrease. This kind of saturation of heavy-element enhancements may explain why enhancements in $(76 \leq \mathrm{Z} \leq 82) / \mathrm{O}$ do not exceed those in $(50 \leq \mathrm{Z} \leq 56) / \mathrm{O}$. Depletion of ${ }^{3} \mathrm{He}$ in the flare volume has been invoked to explain the systematic decrease in ${ }^{3} \mathrm{He} /{ }^{4} \mathrm{He}$ in impulsive SEP events with increasing particle fluences (Reames 1999). With reasonable approximations, the number of energetic ${ }^{3} \mathrm{He}$ ions in space was found to be a significant fraction of the number available in a flare volume. Although the abundance of ${ }^{3} \mathrm{He}$ exceeds that of $Z \geq 50$ ions in the flare plasma, ${ }^{3} \mathrm{He}$ is presumably accelerated preferentially because it resonates with electromagnetic ion cyclotron waves that peak near the ${ }^{3} \mathrm{He}$ gyrofrequency (Temerin $\&$ Roth 1992; Roth \& Temerin 1997) while the acceleration of $Z \geq 50$ ions involves cascading Alfvén turbulence.

The strong coupling between spectra and abundances in impulsive SEP events, seen for both ${ }^{3} \mathrm{He}$ (Mason et al. 2002) and for $Z \geq 50$, seems to argue against a 2-phase process (e.g. Fisk 1978) where a selective heating mechanism determines the enhancements while a separate acceleration mechanism determines the spectra. The observations seem to favor acceleration and enhancement of all ions by a single resonant process, although different wave modes may resonate preferentially with different ions.

\section{CONCLUSIONS}

Element abundances of 2-10 MeV amu ${ }^{-1}$ ions from SEP events show a bimodal pattern reflecting the underlying contributions of impulsive and gradual SEP events that result from two different acceleration mechanisms. Flare-associated impulsive events have enhancements in ions with $Z \geq 50$ by factors of $100-10,000$, relative to coronal abundances while the corresponding enhancements in the shock-accelerated particles from gradual events are only $0.2-20$.

The enhancements in $Z \geq 50$ ions in impulsive events are uncorrelated with $\mathrm{Fe} / \mathrm{O}$ but are strongly correlated with the energy spectral index of $\mathrm{Fe}$, with the Fe fluence, and with the soft X-ray flux from the flare. Smaller events and those with steeper energy spectra have greater heavy element enhancements. This suggests that when the flare energy available for acceleration is small, much of it is absorbed first by rare heavy elements (and ${ }^{3} \mathrm{He}$ ) leaving only enough to produce steep energy spectra for the more abundant ions. As the energy input increases, the supply of heavy elements is depleted and more energy flows into the abundant element species at higher $Q / A$, accelerating them in greater numbers with increasingly hard spectra.

We thank G. M. Mason for helpful discussions of the Mason et al. (2004) results. 


\section{REFERENCES}

Arnaud, M., \& Raymond, J. 1992, ApJ. 398, 394.

Arnaud, M., \& Rothenflug, R. 1985, Astron. and Astrophys. Suppl. 60, 425.

Desai, M. I., Mason, G. M., Mazur, J. E., Dwyer, J. R., Gold, R. E., Krimigis, S. M., Smith, C. W., \& Skoug, R. M. 2003, ApJ 588, 1149.

Fisk, L. A. 1978, ApJ 224, 1048.

Gosling, J. T. 1993, J. Geophys. Res., 98, 18937.

Grevesse, N., \& Sauval, A. J. 1998, Space Sci. Revs. 85, 161.

Kahler, S. W. 1992, Ann. Rev. Astron. Astrophys. 30, 113.

Kahler, S. W. 1994, ApJ, 428, 837.

Kahler, S. W. 2001, J. Geophys. Res. 106, 20947.

Lee, M. A. 1983, J. Geophys. Res., 88, 6109.

Lee, M. A. 1997, in: Coronal Mass Ejections, edited by N. Crooker, J. A. Jocelyn, J. Feynman, Geophys. Monograph 99 (AGU press), 227.

Mazur, J. E., Mason, G. M., Dwyer, J. R., Giacalone, J., Jokipii, J. R., \& Stone, E. C 2000, ApJ 532, L79.

Mason, G. M. et al. 2002, ApJ 574, 1039.

Mason, G. M., Mazur, J. E., \& Dwyer, J. R. 1999, ApJ (Letters) 525, L133.

Mason, G. M., Mazur, J. E., Dwyer, J. R., Jokipii, J. R., Gold, R. E., \& Krimigis, S. M. 2004 ApJ (in press).

Meyer, J. P. 1985, ApJS 57, 151.

Miller, J. A. \& Viñas, A. F. 1993, ApJ 412, 386.

Miller, J. A. 1998, Space Sci. Revs. 86, 79.

Ng, C. K., Reames, D. V., \& Tylka, A. J. 1999, Geophys. Res. Lett. 26, 2145.

Ng, C. K., Reames, D. V., \& Tylka, A. J. 2003 ApJ 591, 461.

Post, D. E., Jensen, R. V., Tarter, C. B., Grasberger, W. H., \& Lokke, W. A. 1977, Atomic Data and Nucl. Data Tables, 20, 397.

Reames, D. V: 1988, ApJ 330, L71.

Reames, D. V. 1995a, Adv. Space Res. 15 (No. 7), 41.

Reames, D. V. 1995b, Revs. Geophys. (Suppl.) 33, 585.

Reames, D. V. 1999, Space Sci. Revs., 90, 413.

Reames, D. V. 2000, ApJ 540, L111.

Reames, D. V. 2002, ApJ 571, L63.

Reames, D. V., Barbier, L. M., von Rosenvinge, T. T., Mason, G. M., Mazur, J. E., \& Dwyer, J. R. 1997, ApJ 483, 515.

Reames, D. V., Dennis, B. R. Stone, R. G., \& Lin, R. P. 1988, ApJ. 327, 998.

Reames, D. V., Meyer, J. P., \& von Rosenvinge, T. T. 1994, ApJS 90, 649.

Reames, D. V., \& McDonald, F. B. 2003, ApJ 586, L99.

Reames, D. V., Ng, C. K., \& Tylka, A. J. 2000, ApJ 531, L83.

Reames, D. V., Ng, C. K., \& Tylka, A. J. 2001; ApJ 548, L233.

Roth, I., \& Temerin, M., 1997 ApJ 477, 940.

Shirk, E. K., \& Price, B. P., 1973, Proc. 13th Int. Cosmic Ray Conf. (Denver) 2, 1474.

Temerin, M., \& Roth, I. 1992, ApJ 391, L105.

Tylka, A. J. 2001, J. Geophys Res. 106, 25333. 
Tylka, A. J., Boberg, P. R., Cohen, C. M. S., Dietrich, W. F., Maclennan, C. G., Mason, G. M., Ng, C. K., \& Reames, D. V. 2002, ApJ 581, L119

Tylka, A. J., Cohen, C. M. S., Deitrich, W. F., Maclennan, C. G., McGuire, R. E., Ng, C. K., \& Reames, D. V. 2001, ApJ 558, L59.

Tylka, A. J., Reames, D. V., \& Ng, C. K. 1999, Geophys. Res. Lett. 26, 2141.

von Rosenvinge, T. T. et al. 1995, Space Sci. Revs. 71, 155. 


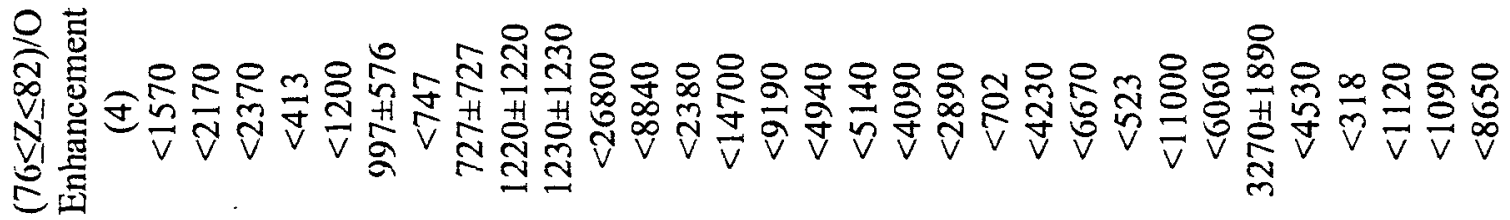

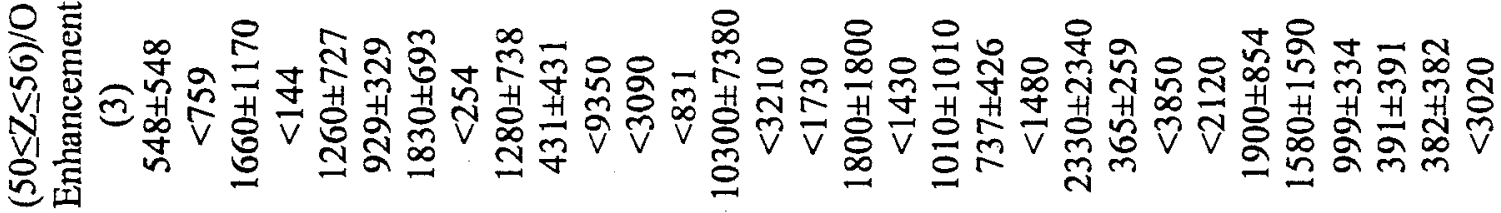

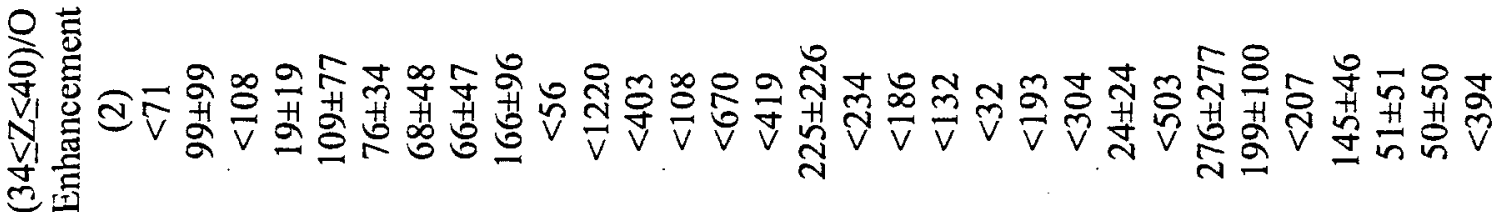

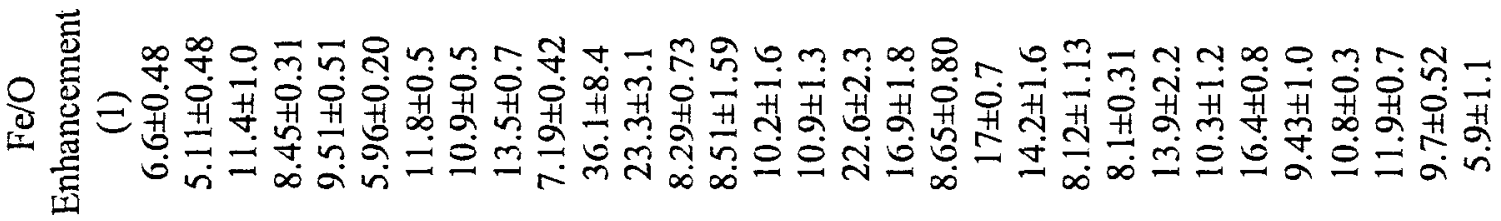
๘ ○

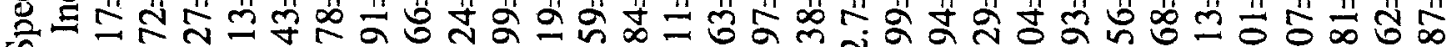

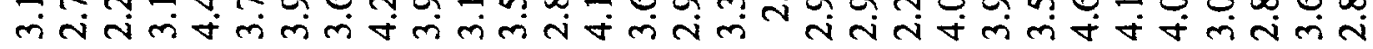

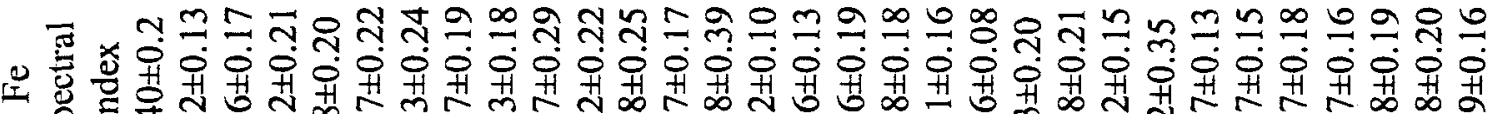
员

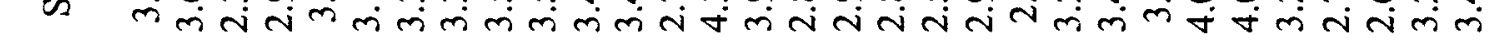
$\bar{z}$

चี

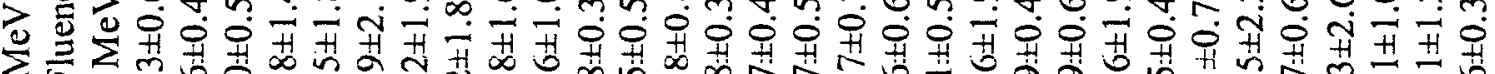

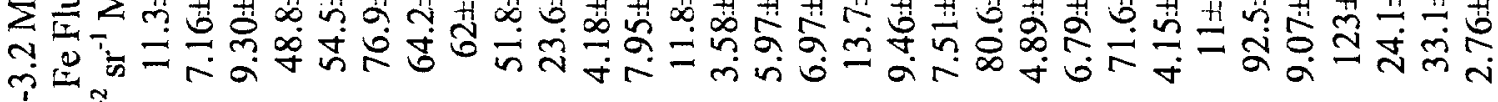
$\stackrel{4}{c^{2}}$

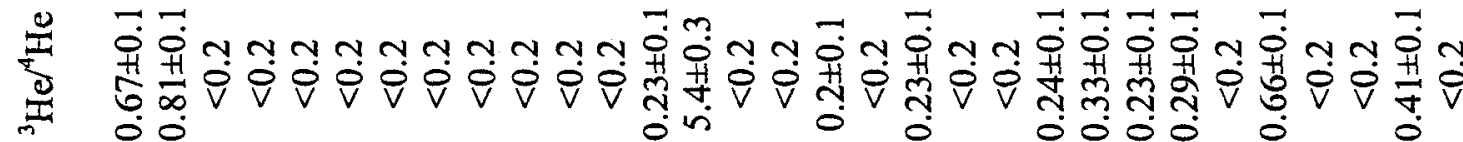

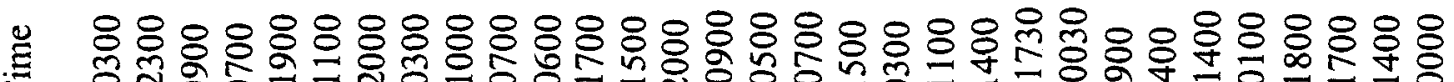

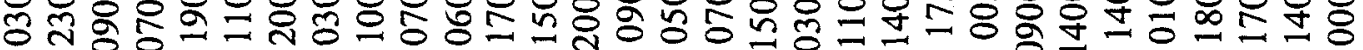

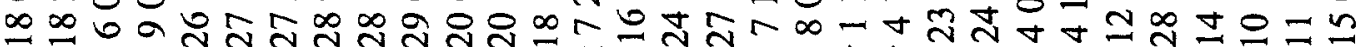

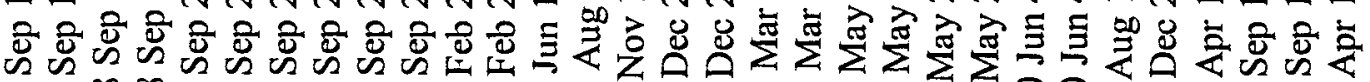
1 $\infty \infty \infty \infty \infty \infty \infty \infty$

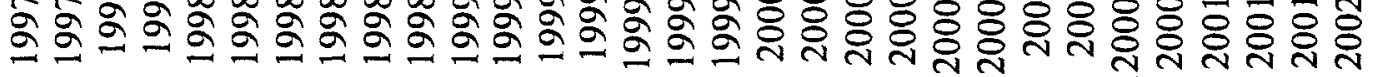

节 - 


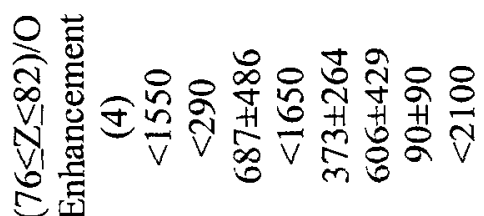

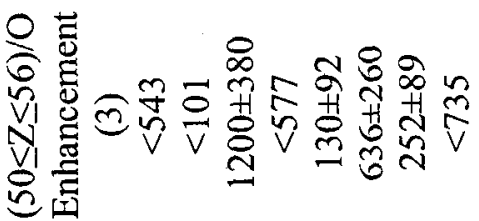

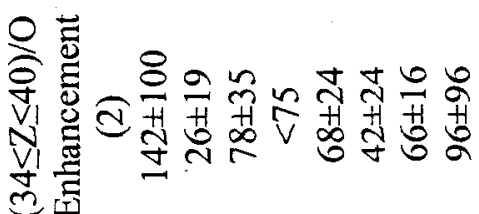

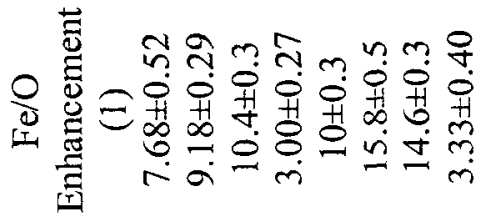

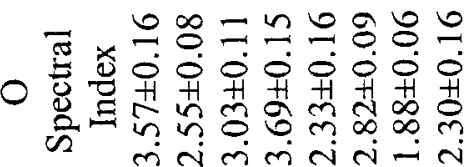

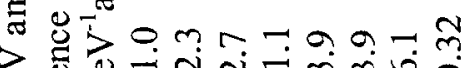

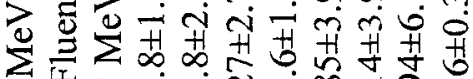

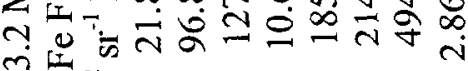
ते

蓄

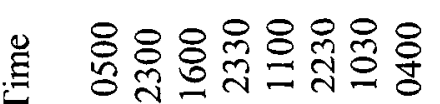

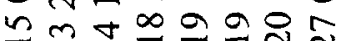

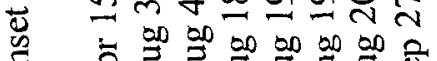

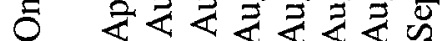

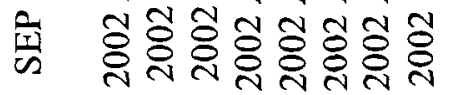

$30 \frac{0}{\circ} \frac{0}{x}$ तิํㅇㅇำ ii $\pi$ 항요웡 0 viv IVIV 造递

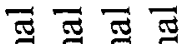
ㅎํㅇㅎํㅇㅎㅇ $\circ \circ \circ$ 0000 恋 $\frac{3}{0} \frac{3}{0} \frac{3}{0}$ $-{ }^{\circ}-T^{\circ} \div$ 芯忌忐忐 $>_{0}>>$ $\sum_{0}^{0} \sum_{0}^{0} \sum_{0}^{0}$ 으으은

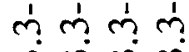
节 Nmm 
Table 2. Large Gradual SEP Events

\begin{tabular}{|c|c|c|c|c|c|c|}
\hline Event & SEP Onset Time & $\begin{array}{c}\text { 2.4-3.2 MeV amu } \\
\text { Fe Fluence } \\
\mathrm{cm}^{-2} \mathrm{sr}^{-1} \mathrm{MeV}^{-1} \mathrm{amu}\end{array}$ & $\begin{array}{c}\mathrm{Fe} / \mathrm{O} \\
\text { Enhancement } \\
\text { (1) }\end{array}$ & $\begin{array}{c}(34 \leq \angle \leq 40) / 0 \\
\text { Enhancement } \\
\text { (2) }\end{array}$ & $\begin{array}{c}(50 \leq 2 \leq 56) / 0 \\
\text { Enhancement } \\
\text { (3) }\end{array}$ & $\begin{array}{c}(76 \leq Z \leq 82) / C \\
\text { Enhancemen } \\
\quad(4)\end{array}$ \\
\hline 1 & 1997 Nov 61400 & $2530 \pm 14$ & $3.15 \pm 0.02$ & $7.7 \pm 1.3$ & $8.2 \pm 3.7$ & $<4.7$ \\
\hline 2 & 1998 Apr 201200 & $6200 \pm 19$ & $2.14 \pm 0.01$ & $2.59 \pm 0.25$ & $0.36 \pm 0.26$ & $<0.52$ \\
\hline 3 & 1998 Aug 242300 & $574 \pm 7$ & $0.393 \pm 0.004$ & $0.275 \pm 0.19$ & $<1.1$ & $<3.0$ \\
\hline 4 & 1998 Sep 231200 & $22.7 \pm 1.1$ & $0.461 \pm 0.022$ & $4.6 \pm 4.6$ & $<36$ & $<10$ \\
\hline 5 & 1998 Sep 301400 & $3110 \pm 21$ & $1.63 \pm 0.01$ & $2.66 \pm 0.58$ & $1.9 \pm 1.4$ & $<2.8$ \\
\hline 6 & 1998 Nov 52200 & $116 \pm 4$ & $0.504 \pm 0.017$ & $<2.5$ & $19 \pm 19$ & $54 \pm 54$ \\
\hline 7 & 1998 Nov 140700 & $1030 \pm 9$ & $3.7 \pm 0.03$ & $7.1 \pm 1.8$ & $10.2 \pm 5.9$ & $9.7 \pm 9.7$ \\
\hline 8 & 1999 Apr 241500 & $68.1 \pm 2.7$ & $0.673 \pm 0.024$ & $11.4 \pm 11.4$ & $<87$ & $<250$ \\
\hline 9 & 1999 May 31200 & $41.5 \pm 1.9$. & $0.155 \pm 0.007$ & $<1.6$ & $<12$ & $<36$ \\
\hline 10 & 1999 Jun 40900 & $651 \pm 7$ & $0.8 \pm 0.009$ & $1.39 \pm 0.70$ & $<2.7$ & $<7.6$ \\
\hline 11 & 2000 Apr 41700 & $353 \pm 6$ & $0.476 \pm 0.007$ & $<0.46$ & $3.5 \pm 3.5$ & $<10$ \\
\hline 12 & 2000 Jun 61900 & $113 \pm 4$ & $0.147 \pm 0.005$ & $<0.75$ & $<5.8$ & $<17$ \\
\hline 13 & $2000 \mathrm{Jul} 141100$ & $20500 \pm 47$ & $3.51 \pm 0.01$ & $22.7 \pm 1.9$ & $4.8 \pm 2.4$ & $<3.4$ \\
\hline 14 & 2000 Sep 121300 & $663 \pm 9$ & $0.241 \pm 0.003$ & $0.18 \pm 0.18$ & $<1.4$ & $<4.0$ \\
\hline 15 & 2000 Nov 82300 & $40100 \pm 109$ & $3.22 \pm 0.01$ & $15.5 \pm 1.8$ & $3.0 \pm 2.1$ & $4.3 \pm 4.3$ \\
\hline 16 & 2000 Nov 240600 & $1120 \pm 10$ & $1.06 \pm 0.009$ & $1.7 \pm 0.7$ & $<1.9$ & $<5.4$ \\
\hline 17 & $2001 \operatorname{Jan} 281800$ & $182 \pm 4$ & $1.83 \pm 0.04$ & $3.0 \pm 3.0$ & $<23$ & $<65$ \\
\hline 18 & $2001 \mathrm{Mar} 251200$ & $24.3 \pm 1.2$ & $0.402 \pm 0.021$ & $4.8 \pm 4.8$ & $<37$ & $<105$ \\
\hline 19 & $2001 \mathrm{Mar} 291200$ & $433 \pm 6$ & $3.36 \pm 0.04$ & $3.7 \pm 2.1$ & $<9.5$ & $<27$ \\
\hline 20 & 2001 Apr 30000 & $4820 \pm 24$ & $2.35 \pm 0.01$ & $4.2 \pm 0.7$ & $2.5 \pm 1.5$ & $<2.4$ \\
\hline 21 & $2001 \mathrm{Apr} 101000$ & $1410 \pm 13$ & $0.887 \pm 0.008$ & $0.49 \pm 0.34$ & $<1.9$ & $<5.3$ \\
\hline 22 & 2001 Apr 151400 & $2630 \pm 17$ & $2.56 \pm 0.017$ & $1.97 \pm 0.74$ & $4.3 \pm 3.1$ & $<6.2$ \\
\hline 23 & 2001 Apr 180400 & $583 \pm 7$ & $1.57 \pm 0.016$ & $0.9 \pm 0.6$ & $<3.5$ & $<10$ \\
\hline 24 & 2001 May 71300 & $178 \pm 3$ & $1.4 \pm 0.02$ & $<1.4$ & $<11$ & $<31$ \\
\hline 25 & 2001 Aug 91900 & $99.4 \pm 2.9$ & $0.335 \pm 0.010$ & $<1.2$ & $<9.2$ & $<26$ \\
\hline 26 & 2001 Aug 160100 & $257 \pm 4$ & $1.34 \pm 0.017$ & $0.93 \pm 0.66$ & $<3.6$ & $<10$ \\
\hline 27 & 2001 Sep 241100 & $6010 \pm 31$ & $0.958 \pm 0.0046$ & $1.39 \pm 0.27$ & $0.39 \pm 0.39$ & $<1.1$ \\
\hline 28 & 2001 Oct 11300 & $1340 \pm 11$ & $0.535 \pm 0.0039$ & $0.58 \pm 0.21$ & $0.56 \pm 0.55$ & $<1.6$ \\
\hline 29 & 2001 Nov 41400 & $5840 \pm 26$ & $2.52 \pm 0.011$ & $4.01 \pm 0.64$ & $3.2 \pm 1.6$ & $<2.3$ \\
\hline 30 & 2001 Nov 170800 & $31.8 \pm 1.6$ & $0.167 \pm 0.010$ & $<2.4$ & $<18$ & $<53$ \\
\hline 31 & 2001 Nov 221800 & $12600 \pm 42$ & $1.09 \pm 0.004$ & $1.78 \pm 0.23$ & $<0.23$ & $<0.66$ \\
\hline 32 & $2001 \operatorname{Dec} 260600$ & $2060 \pm 15$ & $1.51 \pm 0.01$ & $2.24 \pm 0.65$ & $<1.4$ & $<4.1$ \\
\hline 33 & 2001 Dec 301900 & $1090 \pm 9$ & $0.649 \pm 0.005$ & $0.725 \pm 0.32$ & $<1.1$ & $3.2 \pm 3.2$ \\
\hline 34 & $2002 \operatorname{Jan} 101600$ & $71.6 \pm 2.5$ & $0.111 \pm 0.004$ & $<0.64$ & $<4.9$ & $<14$ \\
\hline 35 & $2002 \operatorname{Mar} 160200$ & $299 \pm 5$ & $0.369 \pm 0.007$ & $<0.69$ & $<5.3$ & $<15$ \\
\hline 36 & $2002 \operatorname{Mar} 180600$ & $296 \pm 6$ & $0.717 \pm 0.016$ & $<1.34$ & $<10$ & $<29$ \\
\hline 37 & $2002 \operatorname{Mar} 221400$ & $19.5 \pm 1.3$ & $0.0387 \pm 0.0036$ & $<1.75$ & $<13$ & $<38$ \\
\hline 38 & 2002 Apr 170700 & $116 \pm 3$ & $0.511 \pm 0.015$ & $<1.66$ & $<13$ & $<37$ \\
\hline 39 & 2002 Apr 210200 & $9330 \pm 30$ & $1.3 \pm 0.004$ & $2.59 \pm 0.27$ & $0.43 \pm 0.30$ & $<0.61$ \\
\hline 40 & 2002 May 221200 & $314 \pm 6$ & $0.282 \pm 0.005$ & $1.41 \pm 0.70$ & $<2.7$ & $<7.7$ \\
\hline 41 & 2002 Jul 160900 & $307 \pm 6$ & $0.5 \pm 0.010$ & $1.38 \pm 0.98$ & $15.9 \pm 9.2$ & $<15$ \\
\hline 42 & 2002 Jul 220000 & $331 \pm 5$ & $0.614 \pm 0.008$ & $0.36 \pm 0.36$ & $<2.8$ & $<7.9$ \\
\hline 43 & 2002 Aug 140300 & $72.8 \pm 2.3$ & $0.467 \pm 0.015$ & $1.9 \pm 1.9$ & $<14$ & $<41$ \\
\hline
\end{tabular}




\begin{tabular}{|c|c|c|c|c|c|c|}
\hline & & 2.4-3.2 $\mathrm{MeV} \mathrm{amu}^{-1}$ & $\mathrm{Fe} / \mathrm{O}$ & $(34 \leq \mathrm{Z} \leq 40) / \mathrm{O}$ & $(50 \leq \mathrm{Z} \leq 56) / \mathrm{O}$ & $(76 \leq \mathrm{Z} \leq 82) / \mathrm{O}$ \\
\hline Event & SEP Onset Time & $\begin{array}{c}\text { Fe Fluence } \\
\mathrm{cm}^{-2} \mathrm{sr}^{-1} \mathrm{MeV}^{-1} \mathrm{amu}\end{array}$ & $\begin{array}{c}\text { Enhancement } \\
\text { (1) }\end{array}$ & $\begin{array}{c}\text { Enhancement } \\
\text { (2) }\end{array}$ & $\begin{array}{c}\text { Enhancement } \\
\text { (3) }\end{array}$ & $\begin{array}{c}\text { Enhancement } \\
\text { (4) }\end{array}$ \\
\hline 44 & 2002 Aug 240600 & $1610 \pm 12$ & $1.16 \pm 0.008$ & $1.65 \pm 0.55$ & $<1.4$ & $<4.0$ \\
\hline 45 & 2002 Sep 52300 & $67.2 \pm 2.1$ & $0.495 \pm 0.015$ & $<1.57$ & $<12$ & $<35$ \\
\hline 46 & 2002 Nov 91600 & $898 \pm 7$ & $0.936 \pm 0.008$ & $0.93 \pm 0.46$ & $1.8 \pm 1.8$ & $<5.1$ \\
\hline 47 & 2003 May 280500 & $971 \pm 9$ & $1.38 \pm 0.014$ & $0.98 \pm 0.70$ & $<3.8$ & $<11$ \\
\hline 48 & 2003 Jun 180700 & $92.9 \pm 2.5$ & $0.21 \pm 0.006$ & $<0.67$ & $<5.1$ & $<15$ \\
\hline
\end{tabular}
(1) at $3.3-10 \mathrm{MeV} \mathrm{amu}^{-1}$, relative to coronal $\mathrm{Fe} / \mathrm{O}=0.134$
(2) at 3.3-10 MeV amu ${ }^{-1}$, relative to coronal $(34 \leq \mathrm{Z} \leq 40) / \mathrm{O}=2.22 \times 10^{-5}$
(3) at $3.3-10 \mathrm{MeV} \mathrm{amu}^{-1}$, relative to coronal $(50 \leq \mathrm{Z} \leq 56) / \mathrm{O}=2.90 \times 10^{-6}$
(4) at 3.3-10 MeV amu ${ }^{-1}$, relative to coronal $(76 \leq \mathrm{Z} \leq 82) / \mathrm{O}=1.01 \times 10^{-6}$ 


\section{FIGURE CAPTIONS}

Figure 1. In both panels, particle intensities of $\mathrm{Fe}$ are plotted $v s$. those of $\mathrm{O}$ for all 8-hour intervals in the 9-year study period where $\mathrm{Fe}$ and $\mathrm{O}$ are measurable. The indicated intensity of ACR $O$ forms a lower bound on the $O$ intensity during solar minimum. The symbols used for points in the upper panel indicate the average value of ${ }^{3} \mathrm{He} /{ }^{4} \mathrm{He}$ during each interval, as indicated in the scale to the right of the panel. Symbols in the lower panel denote the $(50 \leq \mathrm{Z} \leq 56) / \mathrm{O}$ abundance ratio shown in the right-hand scale. Lines drawn along $\mathrm{Fe} / \mathrm{O}=1$ and 0.1 , approximately track the locus expected for impulsive and gradual SEP events.

Figure 2. Intensities of various ion species vs. time are compared for 13 impulsive SEP events spanning a wide variety of intensities and element abundances (see text).

Figure 3. The enhancements in the heavy elements abundance ratios $(34 \leq Z \leq 40) / O$, $(50 \leq \mathrm{Z} \leq 56) / \mathrm{O}$, and $(76 \leq \mathrm{Z} \leq 82) / \mathrm{O}$, relative to coronal abundance ratios, are shown $v s$. the corresponding enhancement in $\mathrm{Fe} / \mathrm{O}$ for a sample of 39 large impulsive events.

Figure 4. Abundance enhancements in average "large" impulsive events, relative to coronal abundances, are shown as a function of $Z$ and of $Q / A$ at $\sim 3 \mathrm{MK}$ for the present (solid circles) study and for other elements measured in previous (open circles) studies (see text).

Figure 5. Energy spectra are shown for ${ }^{4} \mathrm{He}, \mathrm{C}, \mathrm{O}, \mathrm{Ne}, \mathrm{Si}, \mathrm{Fe}$, and for ions with $34 \leq \mathrm{Z} \leq 40$ and $50 \leq \mathrm{Z} \leq 56$ in 3 impulsive SEP events with measurable heavy elements.

Figure 6. A cross-plot of the absolute values of the power-law spectral indices of $\mathrm{O}$ and $\mathrm{Fe}$ is shown for the 39 large impulsive SEP events of our study. The least-squares fit line (solid) is shown and the diagonal line (dashed) identifies events with equal $\mathrm{Fe}$ and $\mathrm{O}$ spectral indices. The spectra of Fe tend to be flatter than those of $\mathrm{O}$, especially when both spectra are steep.

Figure 7. The enhancements in the $(34 \leq \mathrm{Z} \leq 40) / \mathrm{O},(50 \leq \mathrm{Z} \leq 56) / \mathrm{O}$, and $(76 \leq \mathrm{Z} \leq 82) / \mathrm{O}$, abundances, relative to the corona, are shown as a function of the Fe spectral index for the large impulsive events where the abundances are well determined. The correlation coefficients and least-squares fit lines are shown.

Figure 8. The enhancements in the $(50 \leq \mathrm{Z} \leq 56) / \mathrm{O}$ abundances are plotted as a function of the 2.4-3.2 MeV amu ${ }^{-1}$ fluence of Fe (left panel) and the GOES soft X ray peak flux (right panel) for the large impulsive events where the abundances are well determined. Least-squares fit lines and the correlation coefficients are shown.

Figure 9. The upper panels show the intensities of three large gradual events as a function of time. Lower panels show the enhancements in the abundance ratios $\mathrm{Fe} / \mathrm{O}$, $(34 \leq Z \leq 40) / O$, and $(50 \leq Z \leq 56) / O$, relative to coronal abundance ratios, $v s$. time.

Figure 10. The enhancements in the heavy elements abundance ratios $(34 \leq Z \leq 40) / O$, $(50 \leq Z \leq 56) / O$, and $(76 \leq Z \leq 82) / O$, relative to coronal abundance ratios, are shown $v s$. the corresponding enhancement in $\mathrm{Fe} / \mathrm{O}$ for a sample of 48 large gradual events. Correlation coefficient $(r)$ and least squares fit line are shown in the lower 
panel. Average enhancements are substantially smaller in these gradual events than in the impulsive events shown in Figure 3. 


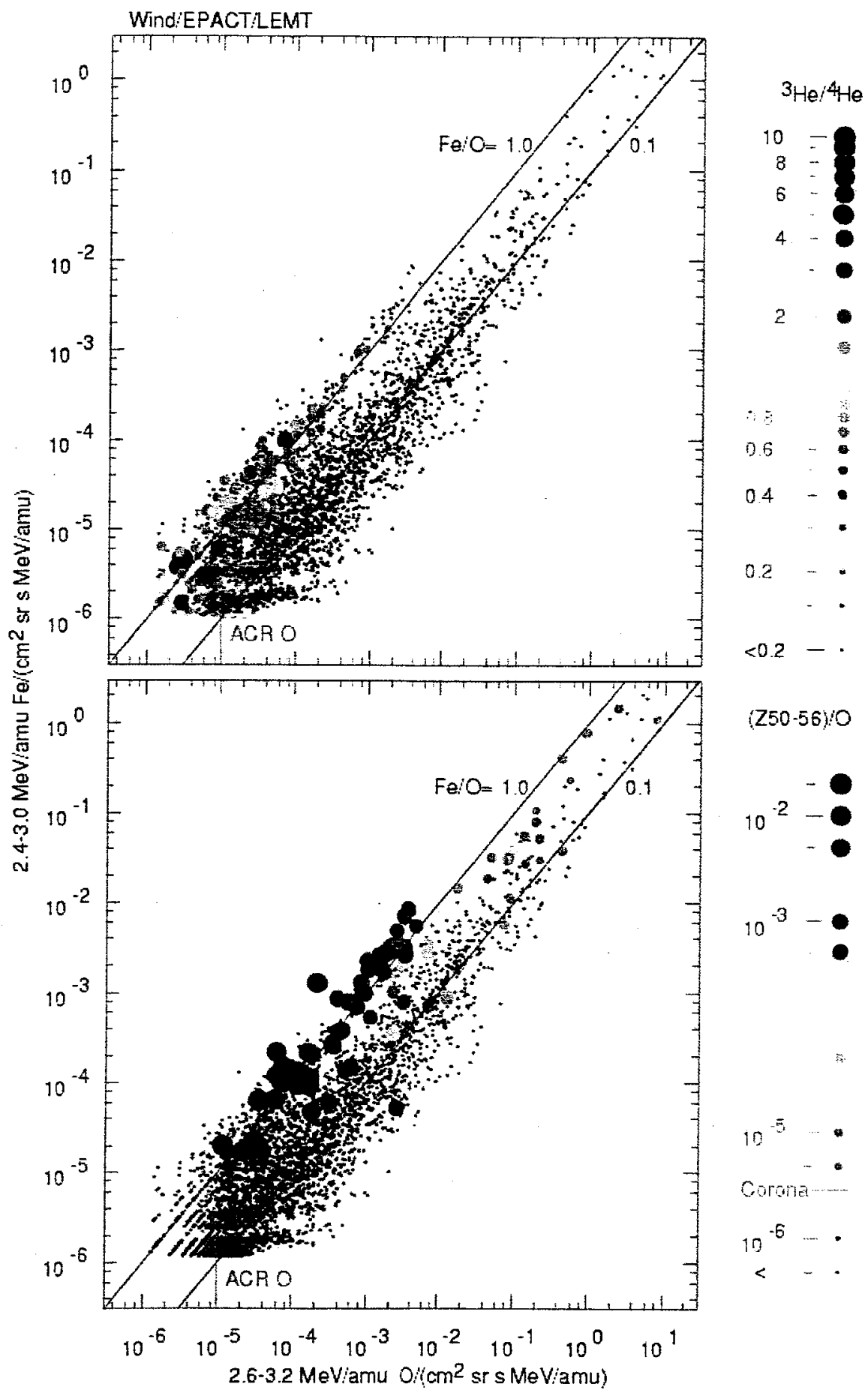

Figure 1. In both panels, particle intensities of $\mathrm{Fe}$ are plotted $v s$, those of $\mathrm{O}$ for all 8-hour intervals in the 9-year study period where $\mathrm{Fe}$ and $\mathrm{O}$ are measurable. The indicated intensity of ACR $O$ forms a lower bound on the $O$ intensity during solar minimum. The symbols used for points in the upper panel indicate the average value of ${ }^{3} \mathrm{He} /{ }^{4} \mathrm{He}$ during each interval, as indicated in the scale to the right of the panel. Symbols in the lower panel denote the $(50 \leq \mathrm{Z} \leq 56) / \mathrm{O}$ abundance ratio shown in the right-hand scale. Lines drawn along $\mathrm{Fe} / \mathrm{O}=1$ and 0.1 , approximately track the locus expected for impulsive and gradual SEP events. 

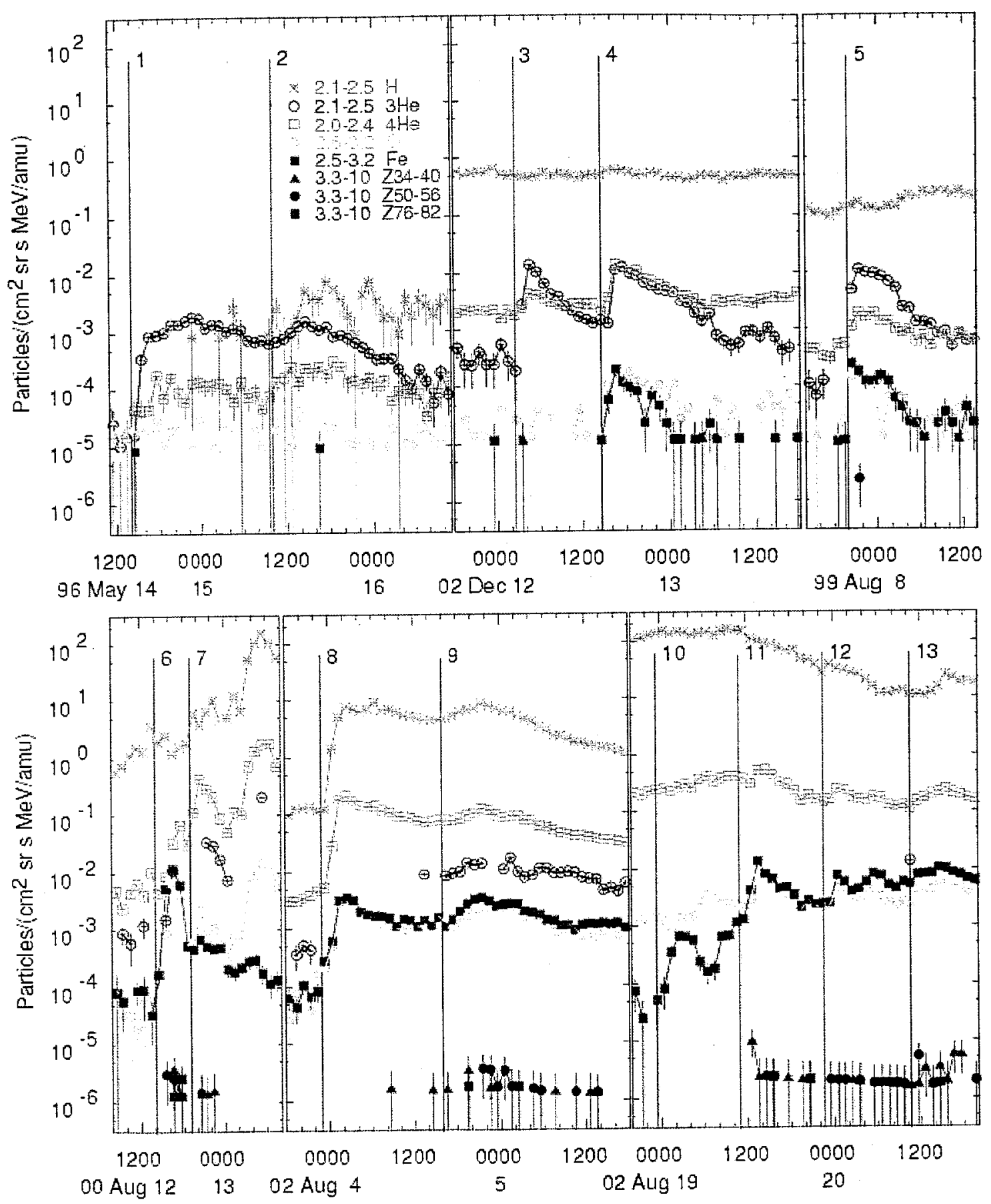

Figure 2. Intensities of various ion species $v s$. time are compared for 13 impulsive SEP events spanning a wide variety of intensities and element abundances (see text). 

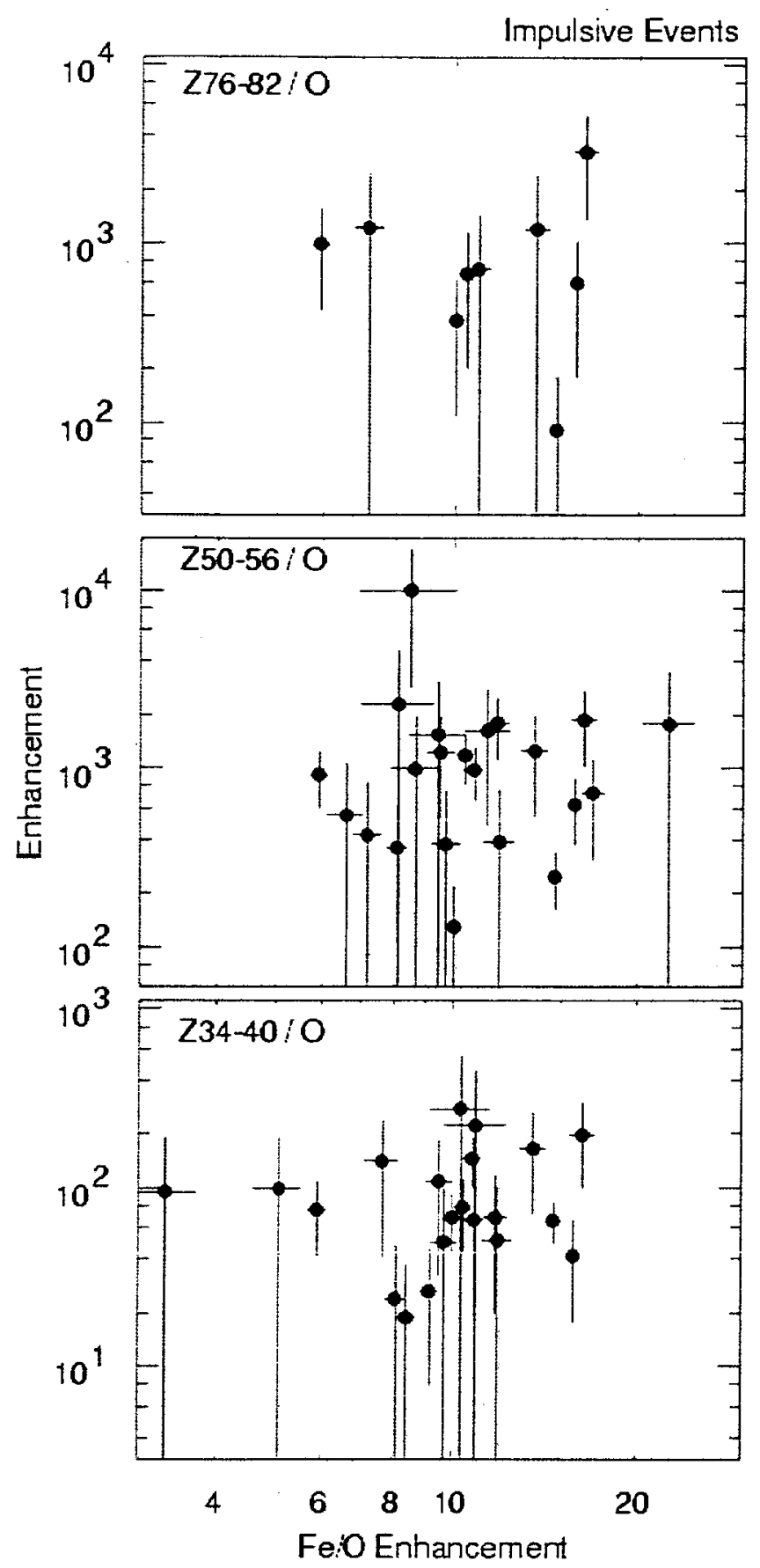

Figure 3. The enhancements in the heavy elements abundance ratios $(34 \leq \mathrm{Z} \leq 40) / \mathrm{O}$, $(50 \leq \mathrm{Z} \leq 56) / \mathrm{O}$, and $(76 \leq \mathrm{Z} \leq 82) / \mathrm{O}$, relative to coronal abundance ratios, are shown vs. the corresponding enhancement in $\mathrm{Fe} / \mathrm{O}$ for a sample of 39 large impulsive events. 


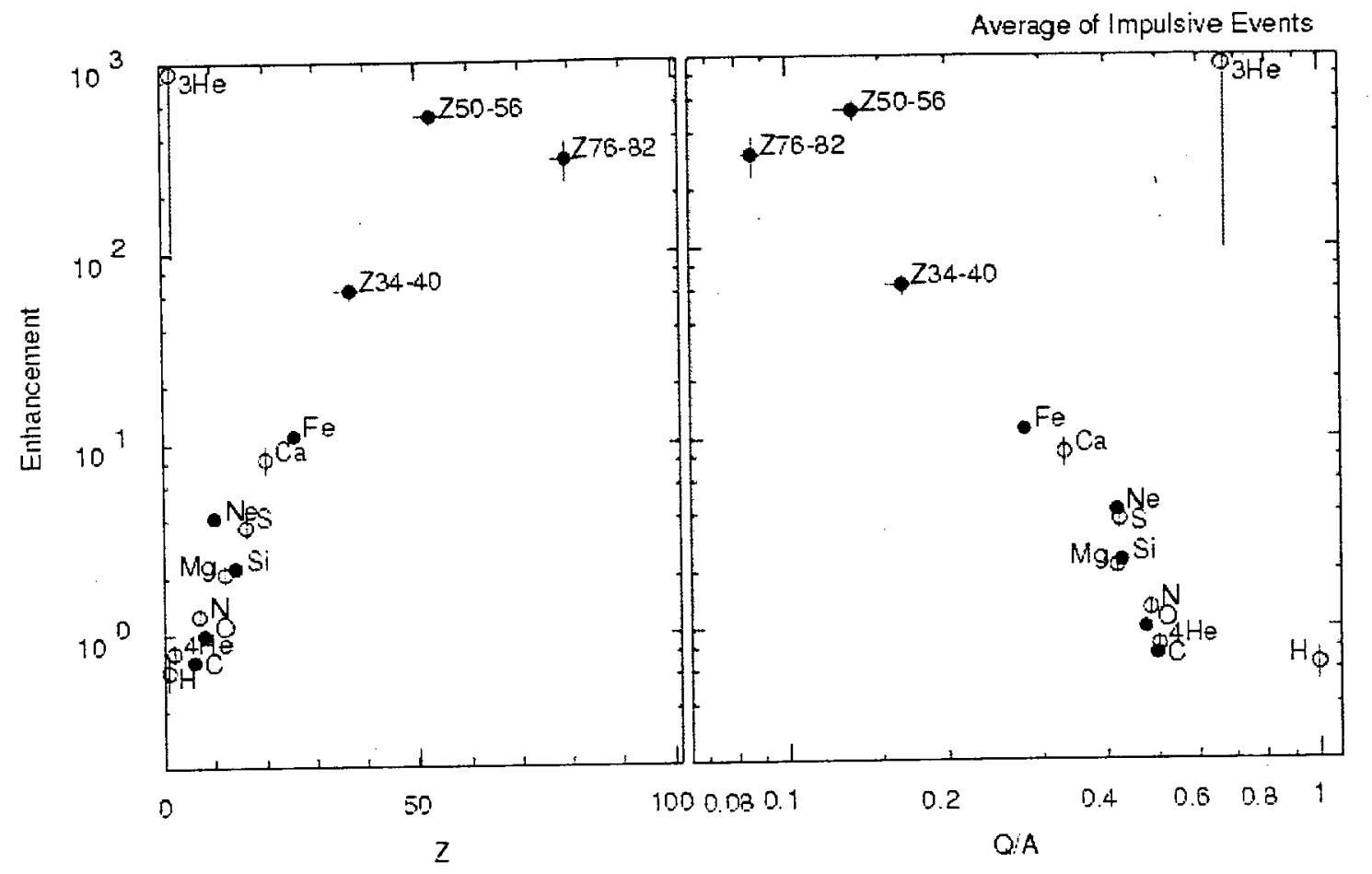

Figure 4. Abundance enhancements in average "large" impulsive events, relative to coronal abundances, are shown as a function of $Z$ and of $Q / A$ at $\sim 3 \mathrm{MK}$ for the present (solid circles) study and for other elements measured in previous (open circles) studies (see text). 


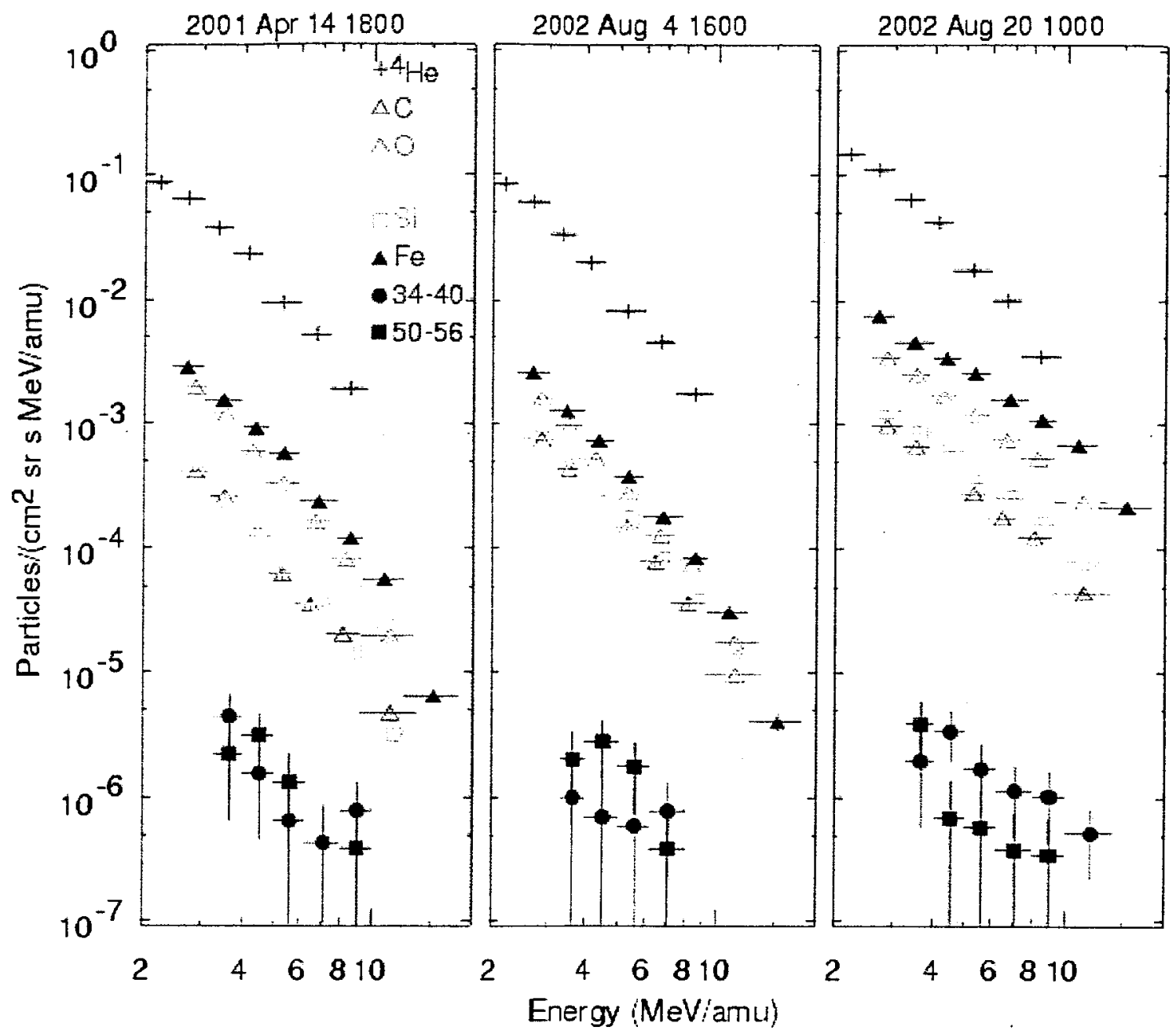

Figure 5. Energy spectra are shown for ${ }^{4} \mathrm{He}, \mathrm{C}, \mathrm{O}, \mathrm{Ne}, \mathrm{Si}, \mathrm{Fe}$, and for ions with $34 \leq \mathrm{Z} \leq 40$ and $50 \leq Z \leq 56$ in 3 impulsive SEP events with measurable heavy elements. 


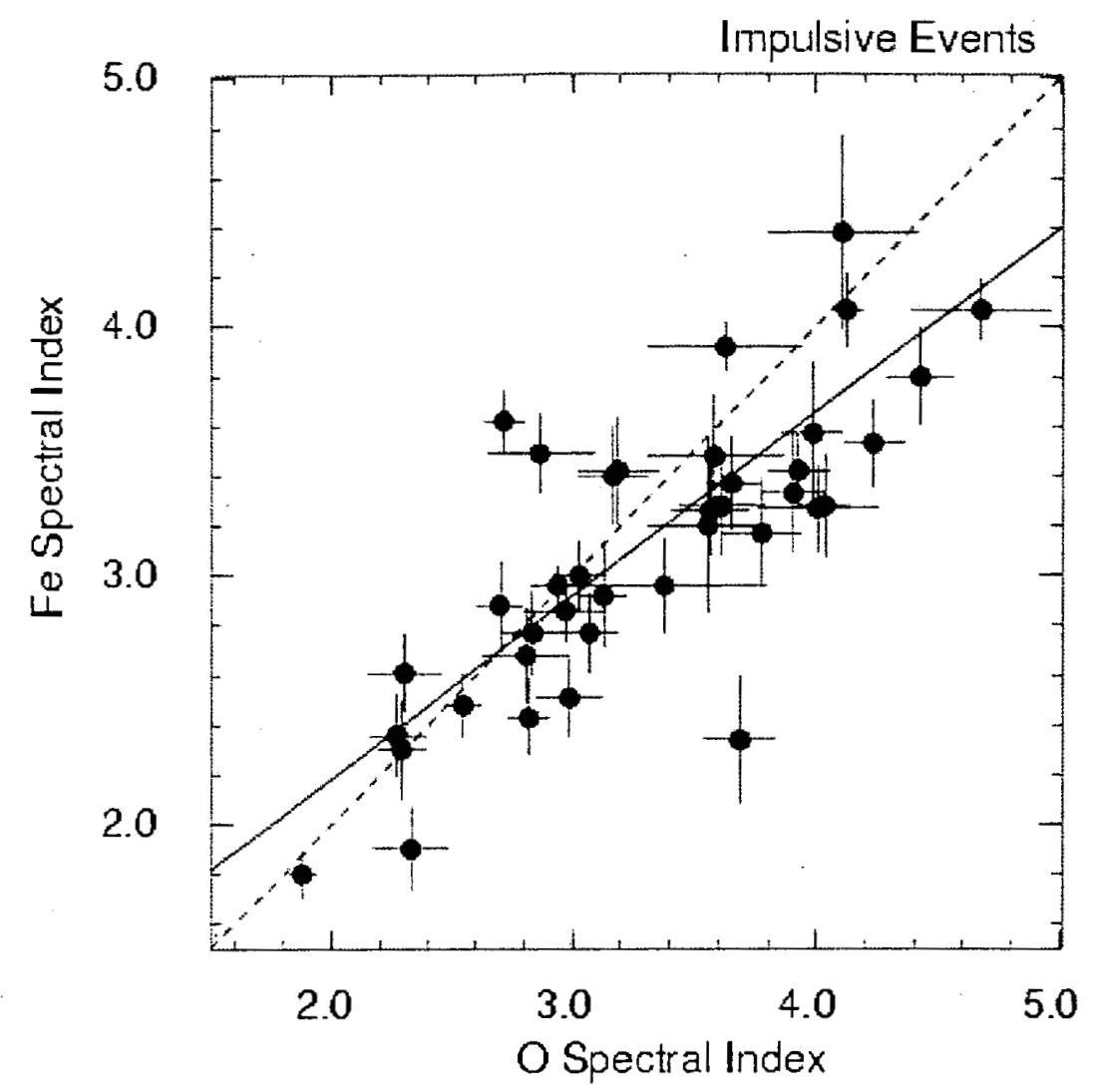

Figure 6. A cross-plot of the absolute values of the power-law spectral indices of $O$ and $\mathrm{Fe}$ is shown for the 39 large impulsive SEP events of our study. The least-squares fit line (solid) is shown and the diagonal line (dashed) identifies events with equal $\mathrm{Fe}$ and $\mathrm{O}$ spectral indices. The spectra of Fe tend to be flatter than those of $\mathrm{O}$, especially when both spectra are steep. 

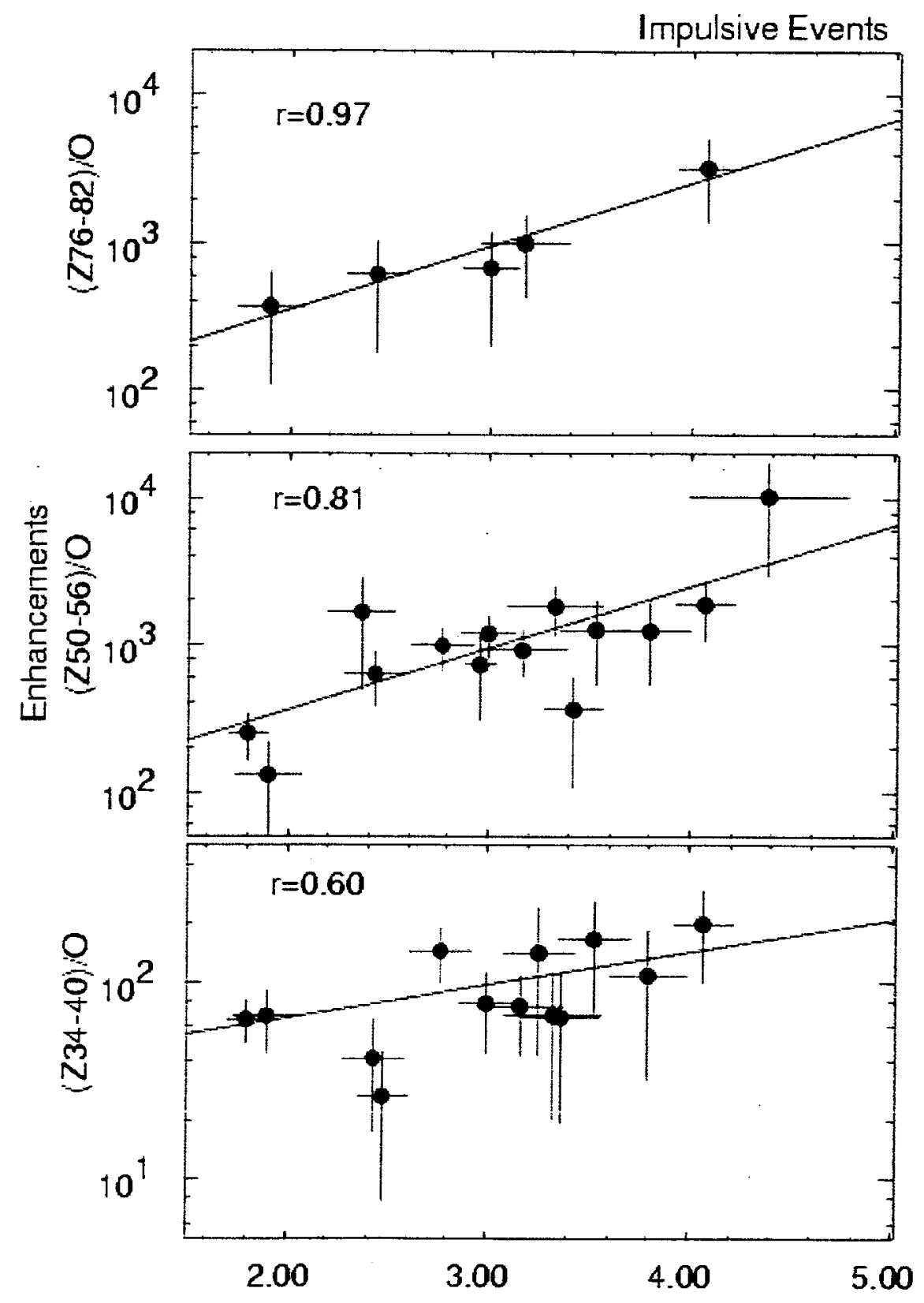

Fe Spectral Index

Figure 7. The enhancements in the $(34 \leq \mathrm{Z} \leq 40) / \mathrm{O},(50 \leq \mathrm{Z} \leq 56) / \mathrm{O}$, and $(76 \leq \mathrm{Z} \leq 82) / \mathrm{O}$, abundances, relative to the corona, are shown as a function of the Fe spectral index for the large impulsive events where the abundances are well determined. The correlation coefficients and least-squares fit lines are shown. 


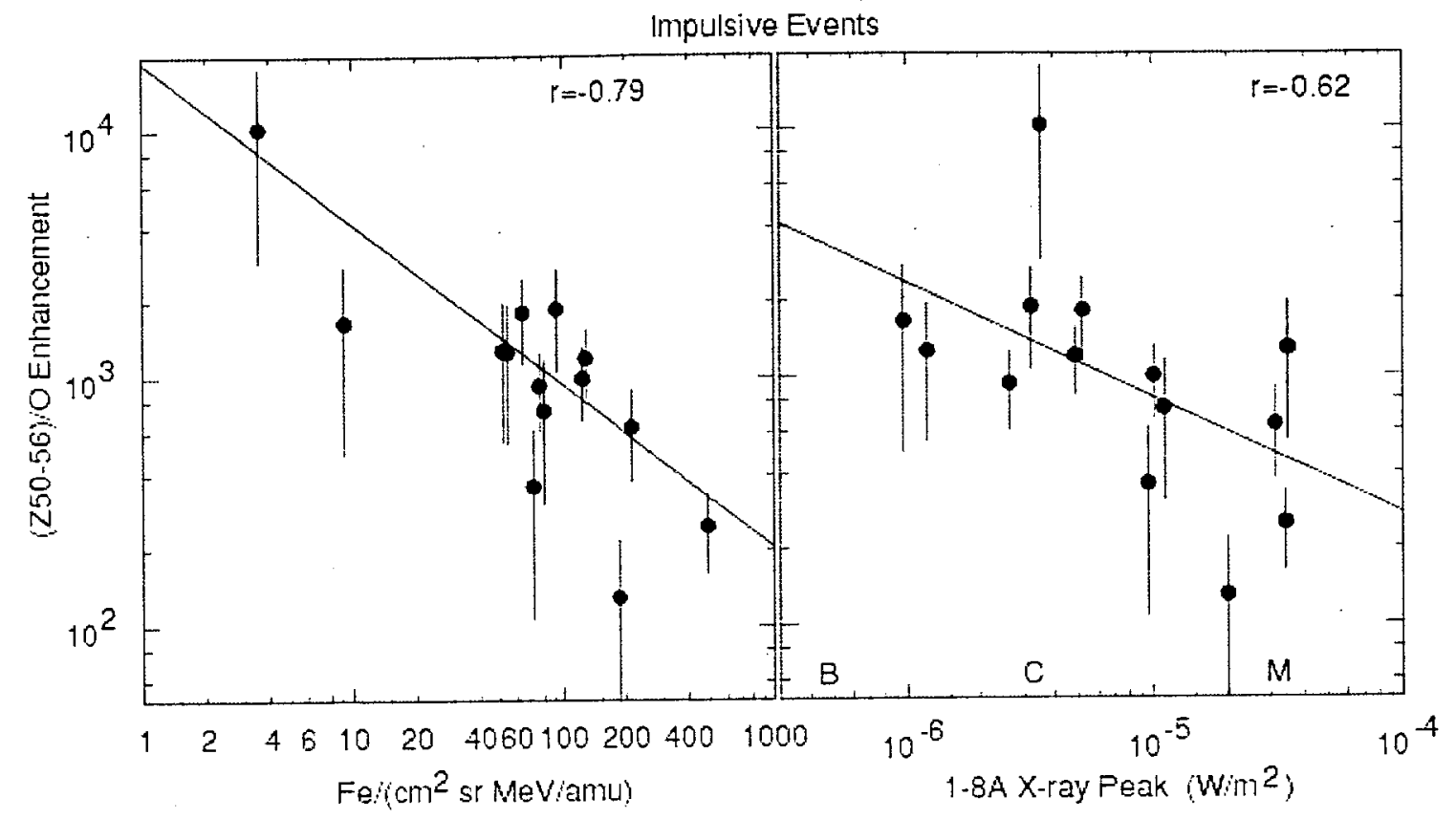

Figure 8. The enhancements in the $(50 \leq \mathrm{Z} \leq 56) / \mathrm{O}$ abundances are plotted as a function of the 2.4-3.2 MeV amu ${ }^{-1}$ fluence of Fe (left panel) and the GOES soft $\mathrm{X}$ ray peak flux (right panel) for the large impulsive events where the abundances are well determined. Least-squares fit lines and the correlation coefficients are shown. 


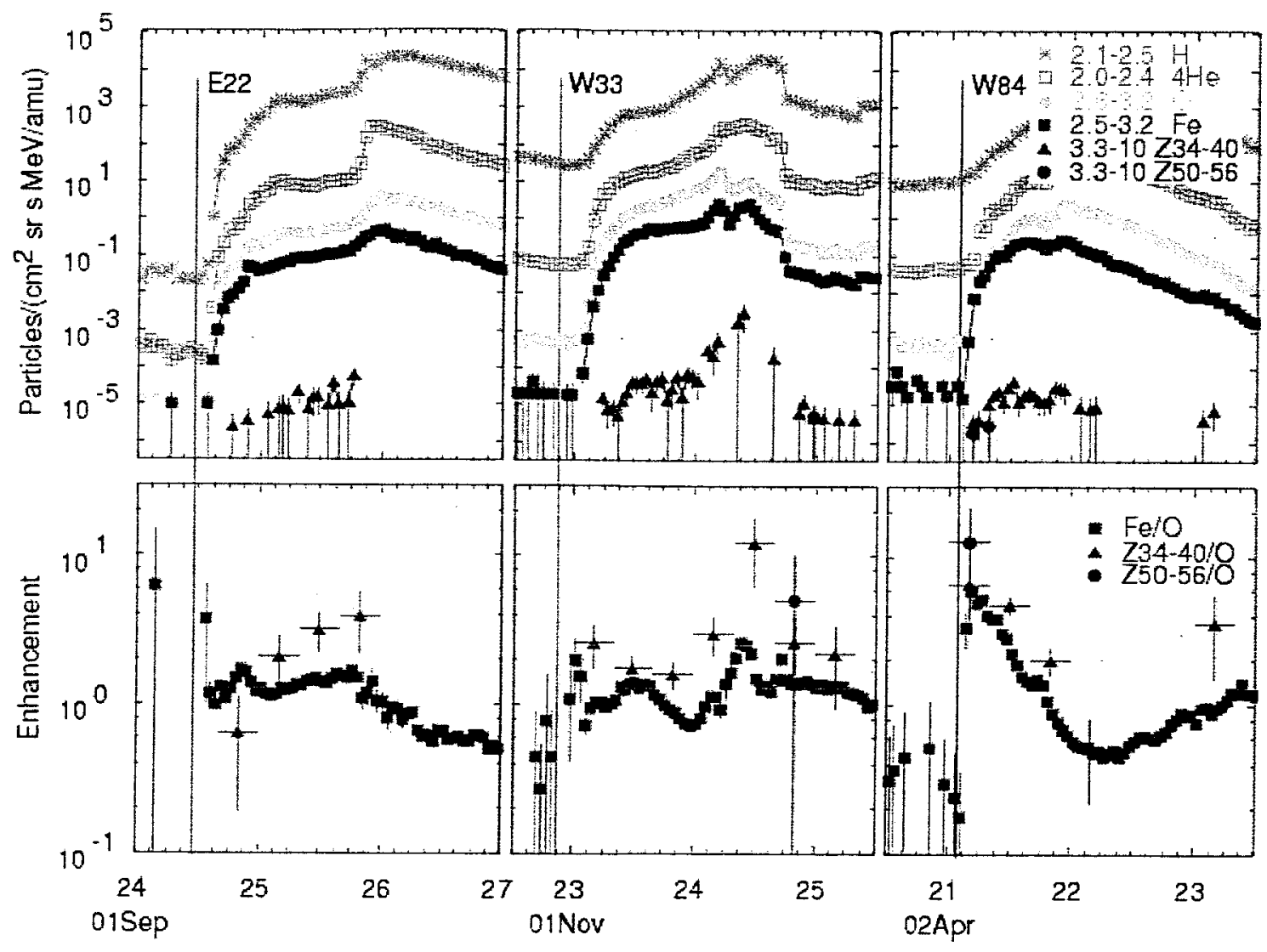

Figure 9. The upper panels show the intensities of three large gradual events as a function of time. Lower panels show the enhancements in the abundance ratios $\mathrm{Fe} / \mathrm{O}$, $(34 \leq \mathrm{Z} \leq 40) / \mathrm{O}$, and $(50 \leq \mathrm{Z} \leq 56) / \mathrm{O}$, relative to coronal abundance ratios, vs. time. 


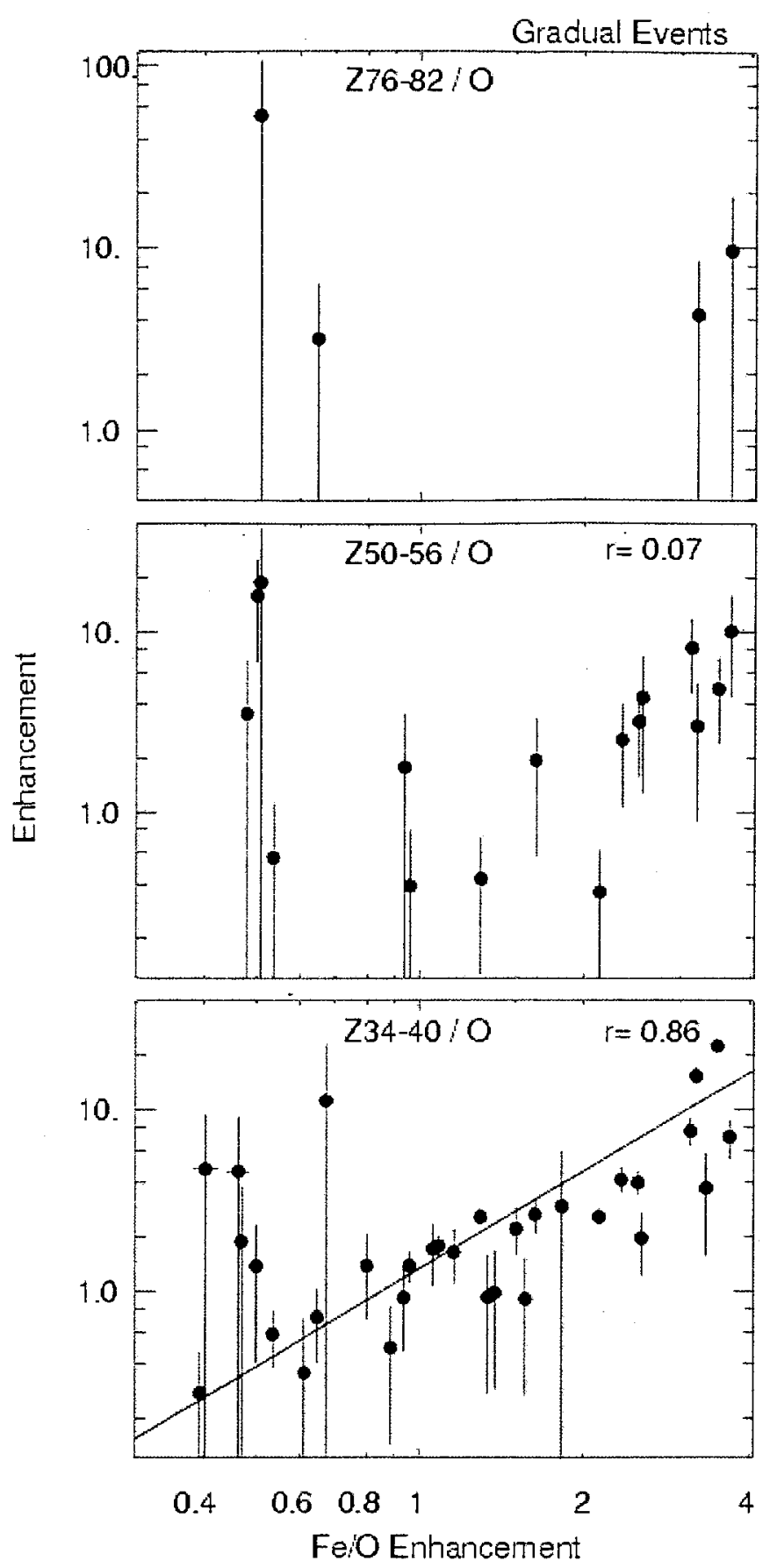

Figure 10. The enhancements in the heavy elements abundance ratios $(34 \leq \mathrm{Z} \leq 40) / \mathrm{O}$, $(50 \leq \mathrm{Z} \leq 56) / \mathrm{O}$, and $(76 \leq \mathrm{Z} \leq 82) / \mathrm{O}$, relative to coronal abundance ratios, are shown $v s$. the corresponding enhancement in $\mathrm{Fe} / \mathrm{O}$ for a sample of 48 large gradual events. Correlation coefficient $(r)$ and least squares fit line are shown in the lower panel. Average enhancements are substantially smaller in these gradual events than in the impulsive events shown in Figure 3. 